\title{
Disentangling star formation and AGN activity in powerful infrared luminous radio galaxies at $1<z<4$
}

\author{
G. Drouart ${ }^{1,2,3}$, B. Rocca-Volmerange ${ }^{3}$, C. De Breuck ${ }^{4}$, M. Fioc $^{3}$, M. Lehnert ${ }^{3}$, N. Seymour ${ }^{2}$, D. Stern ${ }^{5}$, and J. Vernet ${ }^{4}$ \\ ${ }^{1}$ Department of Earth and Space Science, Chalmers University of Technology, Onsala Space Observatory, 43992 Onsala, Sweden \\ 2 International Centre for Radio Astronomy Research, Curtin University, Perth, Australia \\ e-mail: guillaume.drouart@curtin.edu.au \\ 3 Institut d'Astrophysique de Paris, 98bis boulevard Arago, 75014 Paris, France \\ ${ }_{5}^{4}$ European Southern Observatory, Karl Schwarzschild Straße 2, 85748 Garching bei München, Germany \\ 5 Jet Propulsion Laboratory, California Institute of Technology, Mail Stop 169-221, Pasadena, CA 91109, USA
}

Received 3 July 2015 / Accepted 31 May 2016

\begin{abstract}
High-redshift radio galaxies present signs of both star formation and AGN activity, making them ideal candidates to investigate the connection and coevolution of AGN and star formation in the progenitors of present-day massive galaxies. We make use of a sample of 11 powerful radio galaxies spanning $1<z<4$ which have complete coverage of their spectral energy distribution (SED) from UV to FIR wavelengths. Using Herschel data, we disentangle the relative contribution of the AGN and star formation by combining the galaxy evolution code PÉGASE.3 with an AGN torus model. We find that three components are necessary to reproduce the observed SEDs: an evolved and massive stellar component, a submm bright young starburst, and an AGN torus. We find that powerful radio galaxies form at very high-redshift, but experience episodic and important growth at $1<z<4$ as the mass of the associated starburst varies from 5 to $50 \%$ of the total mass of the system. The properties of star formation differ from source to source, indicating no general trend of the star formation properties in the most infrared luminous high-redshift radio galaxies and no correlation with the AGN bolometric luminosity. Moreover, we find that AGN scattered light have a very limited impact on broad-band SED fitting on our sample. Finally, our analysis also suggests a wide range in origins for the observed star formation, which we partially constrain for some sources.
\end{abstract}

Key words. galaxies: active - galaxies: evolution - galaxies: high-redshift - galaxies: star formation - quasars: general galaxies: starburst

\section{Introduction}

High-redshift radio galaxies (HzRG) are among the most luminous objects in the Universe and are the target of a considerable number of investigations aiming to understand galaxy evolution (Miley \& De Breuck 2008, for a review). While powerful radio emission betrays the presence of a supermassive black hole, near-infrared (NIR) images reveal that their host galaxies are amongst the brightest galaxies in the Universe at any redshift (the $K-z$ relation, e.g., Lilly \& Longair 1984; Inskip et al. 2002). Modelling the $K$-band emission suggests that radio galaxies are extremely massive galaxies, $10^{12} M_{\odot}$ (Rocca-Volmerange et al. 2004). Optical images reveal that most radio galaxies present a surprising alignment between the ultraviolet(UV)/optical emission and the radio jet axis (e.g., Chambers et al. 1987; McCarthy et al. 1987; McCarthy 1993; Pentericci et al. 1999, 2001). More generally, the discovery that the mass of the central supermassive black hole is related to its host galaxy and dark matter halo (Magorrian et al. 1998; Gebhardt et al. 2000; Ferrarese \& Merritt 2000; Häring \& Rix 2004) implicates radio galaxies as important objects for understanding the formation and evolution of massive galaxies.

The Spitzer Space Telescope (Werner et al. 2004) provided host galaxy masses of these objects, that place them among the most massive galaxies in the Universe (Seymour et al. 2007). Spitzer also allowed the first characterisation of their mid-infrared (MIR) emission, which showed hot dust close to the supermassive black hole (Haas et al. 2008; De Breuck et al. 2010). In the orientation-based Unification scheme (e.g. Antonucci 1993), the hot dust near central active galactic nuclei (AGN) is completely obscured by material along the line of sight in type $2 \mathrm{AGN}$, and becomes gradually less obscured when transitioning to type 1 AGN (e.g., Leipski et al. 2010; Drouart et al. 2012; Rawlings et al. 2013). Spitzer observations also revealed that high-redshift radio galaxies are preferentially found in denser environments, consistent with galaxy clusters in formation (e.g., Mayo et al. 2012; Galametz et al. 2012; Wylezalek et al. 2013a,b).

The availability of sensitive submm bolometer arrays, such as SCUBA or LABOCA (Holland et al. 1999; Siringo et al. 2009) allowed the first detection of dusty star formation at highredshift through the negative $K$-correction (Blain et al. 1999). Extensive surveys at $z>3$ showed that radio galaxies exhibit high submm flux, indicating vigorous, on-going star formation (e.g., Archibald et al. 2001; Stevens et al. 2003; Reuland et al. 2004), similar to submm galaxies (SMGs, e.g., Borys et al. 2003; Coppin et al. 2006; Weiß et al. 2009) at similar redshift. However, single-dish observations are limited in spatial resolution (typical 20"), and only a handful of sources have been observed with interferometric facilities (De Breuck et al. 2005; Ivison et al. 2012), which makes general conclusions on the entire sample of high-redshift radio galaxies difficult. 
The Herschel Space Observatory (Pilbratt et al. 2010) covers the 60-700 $\mu \mathrm{m}$ range, allowing the first sensitive exploration of the entire infrared (IR) spectral energy distribution (SED) of galaxies in the distant Universe. This part of the SED is of utmost importance because most of the energy of star-forming galaxies is emitted at IR wavelengths. The IR comes from the emission of dust that has absorbed a significant fraction of the UV and optical emission from young stars (e.g., Chary \& Elbaz 2001; Sanders \& Mirabel 1996). AGN emission by dust also contributes mainly in the IR, which can make it difficult to determine the origin of the IR emission. While the submm emission is generally associated with cold dust and therefore with star formation, the complete IR SED allows for a simultaneous characterisation of the AGN and star formation emission (e.g., Tadhunter et al. 2007; Dicken et al. 2009; Mullaney et al. 2011; Feltre et al. 2012; Rosario et al. 2012; Drouart et al. 2014; Leipski et al. 2014). The transition regime between dust heated by the AGN and dust heated by star formation is at $40-60 \mu \mathrm{m}$ and is therefore important to observe because it affects the total infrared emission and characterisation of each component.

As the wavelength coverage of galaxies increased over the past decade, more advanced models were developed to predict the emission of galaxies and AGN for given evolutionary scenarios and/or morphologies. The literature now offers a large number of these models; we only mention here the models using stellar libraries and scenarios of evolution to predict the emission of the integrated emission of the galaxy over a wide wavelength range (e.g., Leitherer \& Heckman 1995; Fioc \& Rocca-Volmerange 1997; Maraston 1998; Bruzual \& Charlot 2003). As the high-redshift infrared observations are becoming increasingly common, the models have extended their coverage to take the dust emission into account (e.g. Leitherer et al. 1999; Burgarella et al. 2005; Maraston 2005; da Cunha et al. 2008; Groves et al. 2008; Noll et al. 2009, for a recent review, see Conroy 2013) The main characteristic of PÉGASE. 3 we use here are to coherently predict attenuated metal-dependent SEDs extended to the dust emission in coherence with metal abundances. The code has been used in RV13 and will be detailed in Fioc et al. (in prep.). Simultaneously, numerous AGN torus models have been developed to characterise nearby AGN. These models predict the UV to far-infrared (FIR) SED as a function of torus geometry, solving the radiative transfer equations. Different approaches have been used to describe the torus properties, particularly to reproduce the $9.7 \mu \mathrm{m}$ silicate feature. The two model types assume either a continuous dust distribution or a clumpy distribution (e.g., Pier \& Krolik 1992; Hönig et al. 2006; Fritz et al. 2006; Nenkova et al. 2008; Stalevski et al. 2012, for a recent review, see Antonucci 2012).

In this paper, we present the first in-depth analysis of the different emission components present for a sample of powerful radio galaxies, handling data continuously covering UV to submm wavelengths. Disentangling the different components of a galaxy is particularly challenging in the UV-NIR domain because several components can contribute simultaneously to the emission and to highly variable proportions. The FIR brings an essential piece of information as, thanks to the energy balance between absorption and emission, constraints on each component are added. We aim in this paper to take advantage of the broad wavelength coverage of both the data and models to reliably disentangle the different components of a sample of highredshift radio galaxies. To investigate the different components, we use two models, one to model the host galaxy and one to model the AGN.
It is difficult to simultaneously characterise the UV-tosubmm SED with AGN and galaxy evolution codes: (i) the multiple components mean an increased number of free parameters; (ii) an efficient fitting procedure is challenging; (iii) a sample of sources needs to be constrained that includes the AGN and host contribution at similar levels; and (iv) homogeneous coverage in wavelength for these samples is necessary. For these reasons, only a few attempts exist in the literature (although the number is increasing thanks to the availability of new techniques and data). Most of these attempts suffer from simplifying one component to study the SED, either the host galaxy or the AGN, depending on the sample or the scientific goals of the study (e.g., Trichas et al. 2012; Feltre et al. 2013; Rocca-Volmerange et al. 2013; Banerji et al. 2014; Karouzos et al. 2014; Leipski et al. 2014; Ciesla et al. 2015).

This paper is structured as follows. We present the sample and the data in Sect. 2. Section 3 describes the fitting procedure and the model used in this analysis. We present the results in Sect. 4 and discuss their implications in Sect. 5. Throughout this paper, we assume the standard concordance cosmological model $\left(H_{0}=70 \mathrm{kms}^{-1} \mathrm{Mpc}^{-1}, \Omega_{\Lambda}=0.7, \Omega_{\mathrm{M}}=0.3\right.$, Spergel et al. 2003).

\section{Sample and data}

To reliably separate the different emission components of radio galaxies, a wide and homogeneous coverage of the SED is of prime importance. The HeRGÉ sample (Herschel Radio Galaxy Evolution sample; Drouart et al. 2014) is ideally suited to estimate the relative contributions of AGN and their host galaxies thanks to the existing Spitzer, Herschel and submm data. We briefly summarise here the criteria selected to build this sample. The radio galaxies were selected to cover homogeneously the radio luminosity-redshift plane, applying the criteria $L^{3 \mathrm{GHz}}>10^{26} \mathrm{WHz}^{-1}$, where $L^{3 \mathrm{GHz}}$ is the total luminosity at a rest-frame frequency of $3 \mathrm{GHz}$ (Table 1; Seymour et al. 2007). To complete our SED coverage from UV to submm, we therefore defined a subsample with the following criteria. Each source (i) must have at least four Herschel detections; (ii) must have at least two broad-band photometric observations bracketing the discontinuity at $4000 \AA$ A restframe; and (iii) the filter response curves should avoid strong emission lines as much as possible. We aim with this selection to obtain more than ten data points from UV to submm wavelengths as homogeneously as possible to allow for a reliable fit. In total, 11 sources remain out of 70 from the HeRGÉ sample (see Fig. 1). In addition, a polarisation measurement at restframe $1500 \AA$ is beneficial, if available, to measure the scattered AGN light. Of our eleven sources, seven have polarisation measurements (from broad-band photometry or spectroscopy, see Table 1 and the detailed discussion in Sect. 4.4). The sources span $1<z<4$, with a median redshift $\langle z\rangle=2.5$. The requirement of Herschel detections biases this sample towards the brightest IR emitters, with $L^{\mathrm{IR}}>$ $4 \times 10^{12} L_{\odot}$ (Drouart et al. 2014). When $L^{\mathrm{IR}}$ is taken into account, these radio galaxies cannot be distinguished from ULIRGs detected by other samples (see Fig. 1). The two highest redshift sources, 4C 41.17 and TN J2007-1316 are extensively analysed in Rocca-Volmerange et al. (2013, hereafter RV13). They had been selected on the basis of their weak AGN contribution in the UV and optical to test the method, which is further improved in this paper. We now extend the sample to 11 sources with a wider range of AGN contributions. 
Table 1. Summarised characteristics of our sample.

\begin{tabular}{|c|c|c|c|c|c|c|c|c|c|}
\hline Name & $\begin{array}{c}z \\
\text { spec. }\end{array}$ & Ref. & $\begin{array}{c}\text { RA } \\
{[\mathrm{J} 2000]}\end{array}$ & $\begin{array}{c}\text { Dec } \\
{[\mathrm{J} 2000]}\end{array}$ & Morph. peculiarities & Radio jet axis & $\begin{array}{c}\log L_{3 \mathrm{GHz}} \\
{\left[\mathrm{W} \mathrm{Hz}{ }^{-1}\right]}\end{array}$ & $\begin{array}{c}\text { Polarisation } \\
{[\%]}\end{array}$ & Ref. \\
\hline $3 C 368$ & 1.132 & $a$ & $18: 05: 06.37$ & $+11: 01: 33.1$ & & & 27.63 & $7.6 \pm 0.9(\mathrm{~V})$ & $g$ \\
\hline $3 \mathrm{C} 4$ & 1 & $b$ & $23: 58: 36$ & $+44: 04: 45.0$ & & misaligned & & & \\
\hline 0324-228 & 1.894 & $c$ & $03: 27: 04.54$ & $-22: 39: 42.1$ & one close companion & 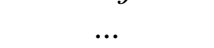 & & $6.5_{-3.3}^{+3.8}(R)$ & $h$ \\
\hline PKS 1138-26 & 2.156 & $c$ & $11: 40: 48.38$ & $-26: 29: 08.8$ & multiple companions & aligned & 28.14 & & \\
\hline MRC 0406-244 & 2.427 & $d$ & 04:08:51.46 & $-24: 18: 16.4$ & multiple components & aligned & 28.11 & $2.5_{-2.5}^{+2.3}(I)$ & $h$ \\
\hline 42 & 2.491 & $e$ & $21: 06:$ & $-24: 05: 09.1$ & two components & misaligned & 27.88 & -2.3 & $\ldots$ \\
\hline & 2.572 & $c$ & 08:30:53.42 & $+19: 13: 15.7$ & e components & aligned & 27.47 & $10.0 \pm 2.0(\mathrm{spec})$ & $i$ \\
\hline $4 \mathrm{C} 2$ & 2.891 & $c$ & $23: 51: 59.20$ & $+29: 10: 29.0$ & multip & aligned & & & \\
\hline USS & 2.92 & $c$ & $09: 45: 32.73$ & $-24: 28: 49.7$ & one $\mathrm{cl}$ & aligned & 27.95 & $6.6 \pm 0.9($ spec $)$ & $i$ \\
\hline $4 \mathrm{C} 41.17^{a}$ & & $c$ & $06: 50: 52.23$ & $+41: 30: 30.1$ & multiple components & aligned & & $<2.4(\mathrm{spec})$ & $j$ \\
\hline TN J2007-1316 ${ }^{a}$ & 3.840 & $f$ & $20: 07: 53.26$ & $-13: 16: 43.6$ & one close companion & $\ldots$ & 27.79 & $\sim 3($ spec) & $k$ \\
\hline
\end{tabular}

Notes. Columns 2 and 3 indicate the spectroscopically confirmed redshifts and their references. Column 6 refers to morphological peculiarities at optical/NIR wavelengths: companion: source close to the object identified as the radio galaxy (few arcsecond) and components: potential substructures when detected in the images. Column 7 indicates the alignment of the radio jet axis compared to the optical/NIR extension when detectable in the images. Column 8 lists the restframe $3 \mathrm{GHz}$ luminosity from De Breuck et al. (2010). The penultimate column indicates the polarisation and the band.

References. (a) Meisenheimer \& Hippelein (1992); (b) Hewitt \& Burbidge (1991); (c) Roettgering et al. (1997); (d) McCarthy et al. (1996); (e) McCarthy et al. (1990); $(f)$ Bornancini et al. (2007); $(g)$ di Serego Alighieri et al. (1989); $(h)$ Buchard (2008); $(i)$ Vernet et al. (2001); $(j)$ Dey et al. (1995); (k) Rocca-Volmerange et al. (2013).

\subsection{Building the SED from broad-band photometry}

We analysed galaxies with multi-wavelength data, therefore the consistency of the photometry throughout the entire spectral range is essential. Specifically, the data from the optical/NIR and FIR domain have very different spatial resolutions. The optical data have sub-arcsec resolution (HST), while the Herschel beam can reach $\sim 35^{\prime \prime}$ (FWHM). For this reason, we report the total flux when we compile photometry from the literature. The total flux was calculated by applying corrections to aperture photometry based on the user manual or primary reference of each corresponding instrument, or by selecting a large aperture, that is, $>30 \mathrm{kpc}\left(>4^{\prime \prime}\right)$ or $64 \mathrm{kpc}\left(\sim 8^{\prime \prime}\right)$.

Based on the filter response and redshift of the source, strong emission lines (i.e., Ly $\alpha,[\mathrm{OII}],[\mathrm{OIII}], \mathrm{H} \alpha \ldots$ ) often fall in a band, increasing the measured flux relative to the pure continuum contribution (by up to $\sim 40 \%$ ). If spectroscopic observations are available for the contaminated bands, we subtracted the estimated flux of the line based on the spectroscopy. All reported fluxes in the tables are corrected and therefore correspond to pure continuum emission for the remainder of this paper (see Tables D.1-D.11). Only MRC 0406-244 does not have the information on line contamination, and fluxes are used as given.

\subsection{Notes on individual sources}

The data set for each source is mainly rich and of high quality. Especially, morphological peculiarities can be isolated and help to understand the overall picture in term of evolution in each source. We report the notes on each source in the Appendix C. We describe the combined high-resolution image and radio contours (when electronically available) in Sect. 5 along with the discussion on the evolutionary status of the galaxy.

\section{Models, fitting procedure, and template libraries}

The broad wavelength coverage from UV to FIR allows us to probe multiple regimes of energy and therefore to better isolate

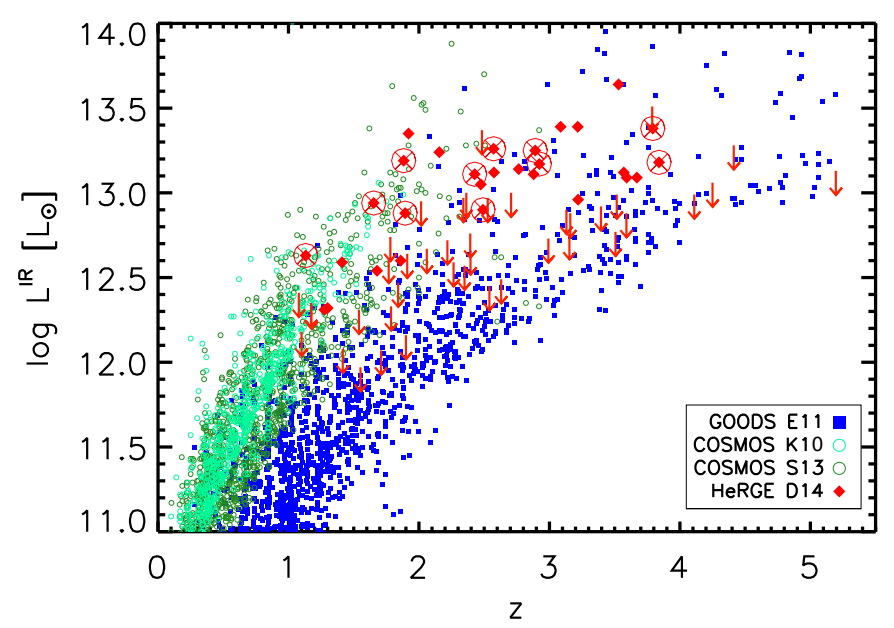

Fig. 1. Total infrared luminosity versus redshift for several samples available in the literature. The light green dots show the COSMOS sample from Kartaltepe et al. (2010), making use of Spitzer data. The blue squares denote the GOODS samples from Elbaz et al. (2011) and the dark green dots represent the selection from Symeonidis et al. (2013), both making use of Herschel data. The red circles indicate the subsample considered in this paper. The red diamonds show the sample of HzRGs presented in Drouart et al. (2014).

emission from specific components of the radio galaxy. The final goal is to disentangle the AGN and stellar emission through SED fitting. We made use of two models, the AGN model of Fritz et al. 2006 and the stellar population model PÉGASE.3 for the host galaxy (Fioc et al., in prep.). We present the fitting procedure, considered models and template libraries for each component (AGN and galaxy) in the following sections. We also explain in detail the creation of the new templates and the assumptions made on both models to limit our total parameter space. 
A\&A 593, A109 (2016)

Table 2. Fixed and fitted parameters for our library of evolved, SB, and AGN templates.

\begin{tabular}{|c|c|c|c|}
\hline & Evolved & SB & AGN \\
\hline \multirow{3}{*}{ Fixed parameters } & Hubble type [Sa, S0, E, E2] & SSP scenario & Opening angle $\theta=\left[40^{\circ}\right]$ \\
\hline & IMF $[$ Kroupa $]$ & IMF [Kroupa] & Radial density profile $[\alpha=0]$ \\
\hline & & & Azimuthal density profile $[\gamma=2]$ \\
\hline \multirow{4}{*}{ Fitted parameters } & Normalisation & Normalisation & $\log _{10} f_{\mathrm{AGN}}^{20 \mu \mathrm{m}}=[-3,-2,-1.7,-1,-0.7,0,0.7,1,2,3]$ \\
\hline & Age, $t_{\text {evolved }}\left[<t_{\text {Universe }, z}\right]$ & Age, $t_{\mathrm{SB}}\left[<t_{\text {evolved }}\right]$ & Inclination $i=[40,50,60,70,80,90]$ \\
\hline & & Column density factor $K=[1,10]$ & Size $Y=[10,60,150]$ \\
\hline & & Initial met. $Z_{\text {init }}=[0,0.0005,0.001,0.005,0.01]$ & Opacity $\tau_{9.7 \mu \mathrm{m}}=[0.1,1,10]$ \\
\hline
\end{tabular}

Notes. We combined the AGN and SB libraries to create hybrid templates. Considered ranges and values in the fitting are reported. In particular, the age of the starburst, $t_{\mathrm{SB}}$, must be younger than the age of the evolved component, $t_{\mathrm{evolved}}$, which in turn must be younger than the age of the Universe at the considered redshift. The single stellar population (SSP) is without infall, has no galactic winds, and is instantaneous.

\subsection{Fitting procedure}

The fitting procedure is presented in RV13. The code allows fitting of up to two stellar components simultaneously based on $\chi^{2}$ minimisation by automatically exploring a library of templates. To adapt the fitting procedure from PÉGASE. 2 to the new code PÉGASE.3, we made the following modifications: (i) we added an estimate of the uncertainties on the fitted quantities; (ii) modified the graphical displaying procedure to include the AGN component; (iii) extended the filter database; and (iv) updated the compatibility with the new outputs of PÉGASE.3.

Adding the AGN component was challenging in the context of the two-component fitting procedure without modifying a large part of the core procedure. We solved this difficulty by creating hybrid templates composed of the sum of the evolving starburst (SB) and AGN components (see Sect. 3.2). The principal problem in adding new components stems from the degeneracies induced by the increase in the number of free parameters. We addressed this specific problem by combining a broad and homogeneous SED coverage (with 12-19 data points) and carefully choosing models and parameters: the self-consistent treatment of the optical and IR emission of the AGN and PÉGASE. 3 models (calculated with radiative transfer codes), enabled us to leave a significant number of parameters free with only limited parameter degeneracies (see Sect. 5 for a discussion of the uncertainties).

Our approach uses grid-model fitting and requires bruteforce calculation through the parameter space. We therefore set some parameters to physically justified values and took representative models to ensure that the code remained executable on a desktop machine in a reasonable computing time. More extensive exploration of the parameter space would require a new fitting algorithm using a Bayesian approach and Monte Carlo sampling of the parameter space, but this is beyond the scope of this paper because it requires a thourough redesign of the core procedure. Although not complete, the coverage of the parameter space is still satisfying, with $>10^{7}$ templates tested for each source.

\subsubsection{PÉGASE model and library}

We used the PÉGASE. 3 code to generate a library of galaxies and starbursts. The new code PÉGASE. 3 is a coherent spectrochemical evolution model predicting simultaneously the metallicity enrichment, the corresponding SED, and the attenuation or emission by dust grain models calculated with radiative transfer Monte Carlo simulations, taking into account scattering and stochastic heating of grains. The continuous synthetic SEDs that extend from the far-UV to submm wavelengths, were built by minimising the input parameter numbers to avoid large degeneracies in the solutions. We characterised each star formation scenario with only four free parameters: star formation rate, initial mass function (IMF), inflows and outflows. We refer to Fioc et al. (in prep.) for a more detailed presentation of the code PÉGASE.3 and its detailed documentation, which is available on the PÉGASE website ${ }^{1}$. In the present version, used hereafter as in RV13, the star formation parameters by spectral types are taken from Le Borgne \& Rocca-Volmerange (2002), fitting the Hubble sequence galaxy properties in the local Universe. These parameters are set to reproduce observed local galaxies from SDSS (Tsalmantza et al. 2009, 2012), including evolution with a formation at $z_{\text {form }}=10$. We focused our library on four galaxy types, E, E2, S0, and Sa to test our sensitivity to the galaxy type when modelling the radio galaxy host. Despite their names, these templates refer to different star formation histories and not to specific morphologies, see Le Borgne \& Rocca-Volmerange (2002).

The starburst (SB) templates are defined differently than the evolved, main component. They are single stellar populations (SSPs), assuming a short formation in one step of time, that is, $1 \mathrm{Myr}$ ( $\delta$ function). SSPs do not take into account any infall or galactic winds. We discuss the effect of this 1 Myr formation assumption in detail in Sect. 4.2. We adopted a Kroupa IMF (Kroupa 2001) and a range of initial metallicities of the gas $\left(Z_{\text {init }}\right)$. RV13 showed that the column density plays a large role in the observed SED.

We explored the same quantities for the evolved population with the SB component, that is, the age and mass of the stellar populations, but we also added the initial metallicity and the column density factor $K$ (see RV13, Sect. 3). Table 2 reports the range of values considered in our library. We here explore the age and mass of the starburst to determine whether the ten times higher column density preferentially found in radio galaxies by RV13 is confirmed for a larger sample. We also note that the fitting procedure requires the evolved component to be younger than the age of the Universe at a given redshift $\left(t_{\text {evolved }}<t_{\text {Universe, } z}\right)$ and the young component to be younger than the evolved component $\left(t_{\mathrm{SB}}<t_{\text {evolved }}\right.$; see also Table 2$)$.

\subsubsection{AGN model and library}

The AGN component was incorporated using the AGN model from Fritz et al. (2006). To briefly describe this model: a central,

1 www2.iap.fr/pegase 
point-like source emits an SED composed of a sum of power laws. The torus is defined in spherical coordinates by its geometry (size and opening angle), dust profile density (radial and azimuthal), opacity at $9.7 \mu \mathrm{m}$ and inclination with respect to the observer. The torus is divided into cells assuming an axisymmetric geometry and the radiative transfer equations are solved using the $\Lambda$-iteration technique (see Fritz et al. 2006, for a complete description of the model). Feltre et al. (2012) showed that the Fritz et al. (2006) model is directly comparable to the clumpy model from Nenkova et al. (2008) for a certain set of parameters (only partially covered in our current library).

We adopted the Fritz et al. (2006) model for the present study, leaving the comparison with clumpy models for further publications. Moreover, a precise characterisation of the torus properties is beyond the scope of this paper, because it requires spectroscopy around the silicate feature at $9.7 \mu \mathrm{m}$ restframe. We focus on the largest impact parameters, that is, the size, the opacity, and the inclination of the torus. The size, $Y$, is the ratio $R_{\max } / R_{\min }$, where $R_{\min }$ is set as the sublimation radius of the dust (which, in turn depends on the luminosity of the central source). The opacity, $\tau_{9.7 \mu \mathrm{m}}$, corresponds to the integrated opacity at $9.7 \mu \mathrm{m}$ along the equatorial axis $\left(i=90^{\circ}\right)$. The dust density (radial and azimuthal) of the torus is calculated with respect to the size, opacity and profile chosen by the user. These values were set to their default values here, radially constant and azimuthally decreasing as the square of the altitude (they also have a limited effect on the SED). The inclination, $i$, is the orientation of the torus with respect to the observer. An edge-on view corresponds to $i=90^{\circ}$, and $i=0^{\circ}$, corresponds to a face-on view. The opening angle, $\theta$, was set to $\theta=40^{\circ}$, as suggested by previous studies (e.g., Barthel 1989; Drouart et al. 2012). In practice, all inclinations $i<50^{\circ}$ present a line of sight free of dust and therefore similar SEDs. To save computing time but include the possibility of a type $1 \mathrm{AGN}$, we only used $i=40^{\circ}$ as a type 1 AGN template. Table 2 presents all the assumed values for the fixed and fitted parameters. The objective of this selection is to explore typical torus configurations and evaluate how our method handles the addition of this third (AGN) component.

\subsection{Hybrid AGN-SB component}

We created hybrid templates, corresponding to the sum of the SB templates produced by PÉGASE.3 and an AGN torus. For each evolving SB template, we normalised an AGN torus (for a given inclination $i$, size $Y$, opacity $\tau_{9.7} \mu \mathrm{m}$ ) to a fraction of the total flux at $20 \mu \mathrm{m}$ following the equation:

$F_{\text {hybrid }}(t)=F_{\mathrm{SB}}(t)+F_{\mathrm{AGN}}\left(i, Y, \tau_{9.7 \mu \mathrm{m}}\right) \times f_{\mathrm{AGN}}^{20 \mu \mathrm{m}}$,

where $F_{\text {hybrid }}$ is the sum of the AGN and the SB templates, $F_{\mathrm{SB}}$ is the SB template, $F_{\mathrm{AGN}}$ is the AGN template and $f_{\mathrm{AGN}}^{20 \mu \mathrm{m}}$ is the relative fraction of the AGN flux compared to the SB template at $20 \mu \mathrm{m}^{2}$ (see Table 2). We performed a step-wise $\chi^{2}$-minimisation that permitting determining of intermediate solutions and checking for degeneracies. In total, $>10^{7}$ templates were fitted for each source.

\subsection{Uncertainties of parameters, $68 \%$ confidence intervals}

Calculating uncertainties on the parameters can be challenging for a multi-dimensional analysis. However, as we performed

\footnotetext{
2 We evaluated $f_{\mathrm{AGN}}^{20 \mu \mathrm{m}}$ with an idealised filter centred on $20 \mu \mathrm{m}$ of $4 \mu \mathrm{m}$ bandwidth to minimise effects from sharp gradients and/or emission and absorption lines (i.e. PAHs).
}

Table 3. Best $\chi^{2}$ for one (evolved only), two (evolved and starburst), and three (evolved, starburst, and AGN) components.

\begin{tabular}{lccc}
\hline \hline Name & 1 comp. & 2 comp. & 3 comp. \\
\# free parameters & 2 & 6 & 10 \\
\hline 3C 368 & $3615[17]$ & $101[13]$ & $73[9]$ \\
3C 470 & $4000[12]$ & $71[8]$ & $32[4]$ \\
MRC 0324-228 & $2152[11]$ & $57[7]$ & $25[3]$ \\
PKS 1138-262 & $14896[14]$ & $834[10]$ & $79[6]$ \\
MRC 0406-244 & $1208[14]$ & $65[10]$ & $19[6]$ \\
MRC 2104-242 & $167[10]$ & $20[6]$ & $9[2]$ \\
USS 0828+193 & $5235[12]$ & $619[8]$ & $36[4]$ \\
4C 28.58 & $247[13]$ & $87[9]$ & $29[5]$ \\
USS 0943-242 & $250[13]$ & $16[9]$ & $5[5]$ \\
4C 41.17 & $491[16]$ & $39[12]$ & $11[8]$ \\
TN J2007-1316 & $148[13]$ & $73[9]$ & $9[5]$ \\
\hline
\end{tabular}

Notes. We also indicate the number of degrees of freedom (d.o.f.) in brackets, d.o.f. $=N-p-1$ where $N$ is the number of data points and $p$ is the number of free parameters.

brute-force calculation through our parameter space, therefore each fit possesses a $\chi^{2}$ value, that indicates the goodness of fit. We can therefore consider the $\chi^{2}$ distribution to gain insights into the confidence interval of the adopted free parameters. We used the description from Numerical recipes (Press et al. 1992, Chap. 15) to estimate the uncertainties on the parameters, using the complementary incomplete gamma function to calculate the corresponding $\Delta \chi^{2}$ corresponding to a $68.3 \%$ confidence interval. For our ten free parameters, this corresponds to $\Delta \chi^{2}=11.5$. We defined the confidence interval as $\chi_{\min }^{2}+\Delta \chi^{2}$ (see Figs. D.1-D.10 and Table 4).

The uncertainties on stellar masses and luminosities were estimated with a different technique because they are directly expressed from the normalisation of the different components. We considered the extreme values of each mass within the allowed $68 \%$ confidence interval of all other parameters and defined this as the lower and upper uncertainties. For the AGN luminosity we added the uncertainty from the AGN fraction $\left(f_{\mathrm{AGN}}^{20 \mu \mathrm{m}}\right)$ in quadrature and the normalisation of the hybrid component. Finally, we add a special caution of interpreting these uncertainties. They only represent the statistical uncertainties on the parameters induced from the noise in the observations and do not take into account potential systematics associated with the model choices and assumptions (see Appendix A for a more extensive discussion of uncertainties and parameter calculation).

\section{Results}

We performed the SED fitting described in the previous sections on the 11 sources in our sample, taking one (evolved component only), two (evolved and starburst), and three (evolved, starburst, and AGN) components into account. Table 3 shows the best $\chi^{2}$ depending on the number of components. The addition of extra components clearly dramatically increases the quality of the fit. This improvement is particularly strong from one to two components and is still significant from two to three components (amelioration by a factor superior to two). We therefore only consider the three-component fit in the remainder of this paper. Table 4 summarises the results of the fitting, including their associated $(68 \%)$ uncertainties. Figure 2 shows an example of the best-fit model for $4 \mathrm{C} 41.17$, while the results for the remaining the sample are presented in Figs. D.1-D.10. We present 

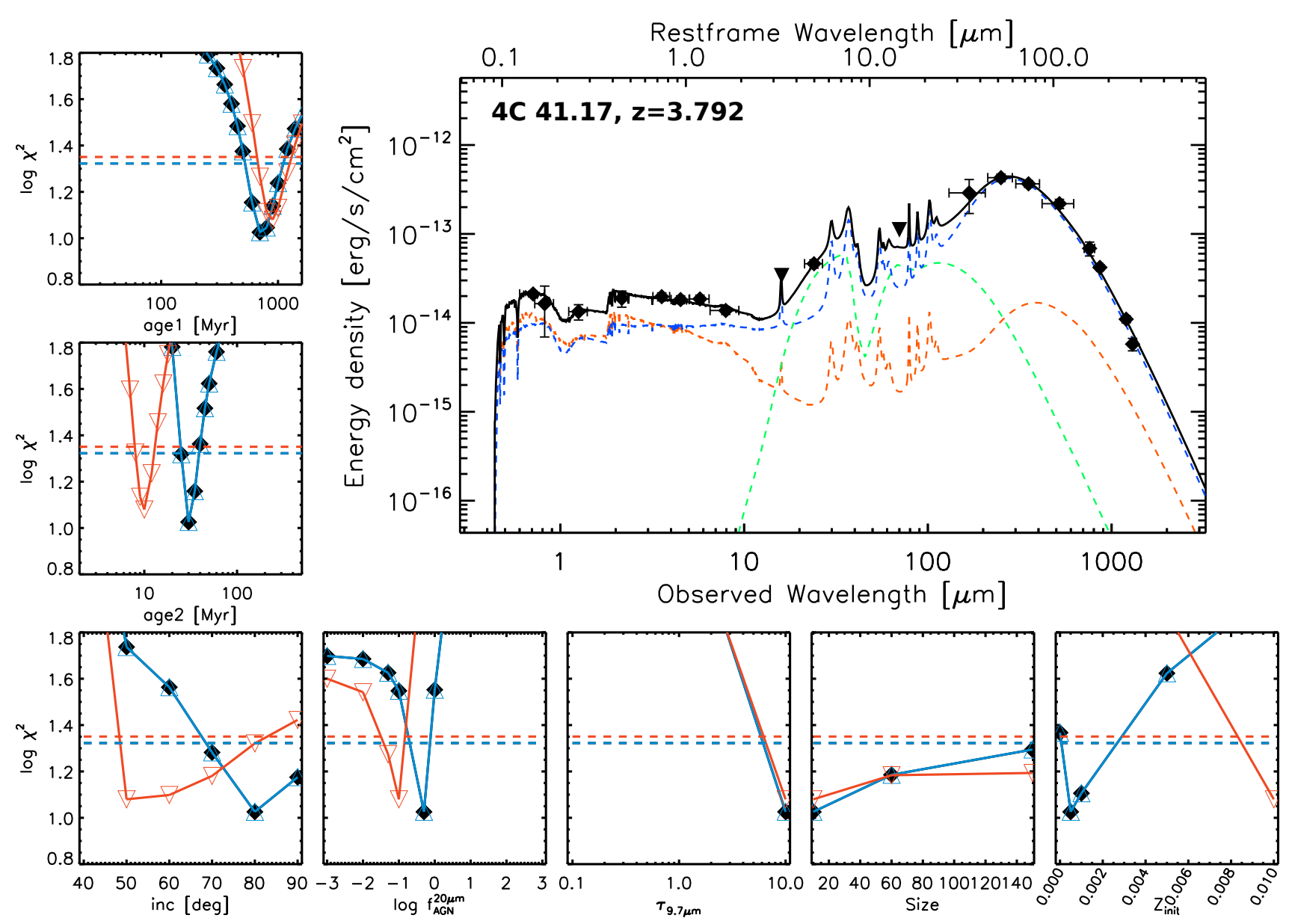

Fig. 2. Best fit for $4 \mathrm{C} 41.17(z=3.792)$. The orange, blue and green dashed lines symbolise the evolved, starburst, and AGN components, respectively. The sum of the components is the dark line, the black diamonds correspond to broad-band photometry, the vertical lines to the $1 \sigma$ uncertainties and horizontal lines to the FWHM of the filters. The downward triangles represent the $3 \sigma$ upper limits. The insets show the $\chi^{2}$ distribution for seven of the free parameters of the fit. From top left to bottom right: age of the evolved component (orange line in the SED), age of the starburst (blue line in the SED), inclination of the AGN torus (green line in the SED), fraction of the AGN at $20 \mu$ m, equatorial opacity of the torus, size of the torus and initial metallicity of the starburst. Black, blue and red indicate the three different approaches we used to estimate the polarisation effect, without, with the lowest and the highest contamination, respectively. We note that in this case the lowest polarisation contribution is zero, hence the black and blue lines are superposed. The horizontal coloured lines correspond to the $68 \%$ confidence interval described in Sect. 3.3.

here the overall trends on the different components for the entire sample. Overall, the fittings are satisfying for all sources even if some discrepancies are still observed (see Sect. 5.1). It is important to note that three components are necessary to reproduce the SED of the powerful radio galaxies in this sample, and each appears to be dominating at a particular wavelength range.

\subsection{Evolved component}

We call the oldest stellar component the evolved component $(>0.5 \mathrm{Gyr})$, which can be defined as the radio galaxy host. Figure 3 shows the mass of the evolved stellar population as a function of redshift (red points). The host galaxy appears massive at $z=4$, and this evolved massive component is present in every source throughout our redshift range $\left(M_{\text {stel }}>\right.$ $10^{11} M_{\odot}$, except for MRC $0324-228$ with $\left.10^{10.7} M_{\odot}\right)$. Previous studies have shown that the most powerful radio galaxies present exceptionally high masses (e.g. De Breuck et al. 2002; Rocca-Volmerange et al. 2004; Seymour et al. 2007). This places powerful radio galaxies at the top of the galaxy mass distribution seen in large field galaxy samples (e.g., Marchesini et al. 2009; Ilbert et al. 2013). Interestingly, these masses are also comparable to SMGs at similar redshift (e.g., Borys et al. 2003). This suggests that powerful radio galaxies are drawn from the same sample of massive galaxies, formed at high-redshift $(z>6)$ on a relatively short timescale. Similarly as in RV13, the evolved component clearly dominates the Spitzer photometry, consistent with the shift of the $1 \mu \mathrm{m}$ peak of the most evolved early-type galaxies.

The right panel of Fig. 3 confirms this result because all red points are clustered along the black lines (plain and dashed), consistent with an early formation epoch. We also note that the four Hubble types used in our analysis provide similar $\chi^{2}$ values within the $68 \%$ confidence ranges. We therefore conclude that low-resolution broad-band photometry alone cannot constrain the exact star formation history of the galaxies, but still favours a formation at early times in the Universe. We emphasise that the ages determined by our method are well constrained, as illustrated in Figs. D.1-D.10 where the best $\chi^{2}$ solutions are located in deep minima. 

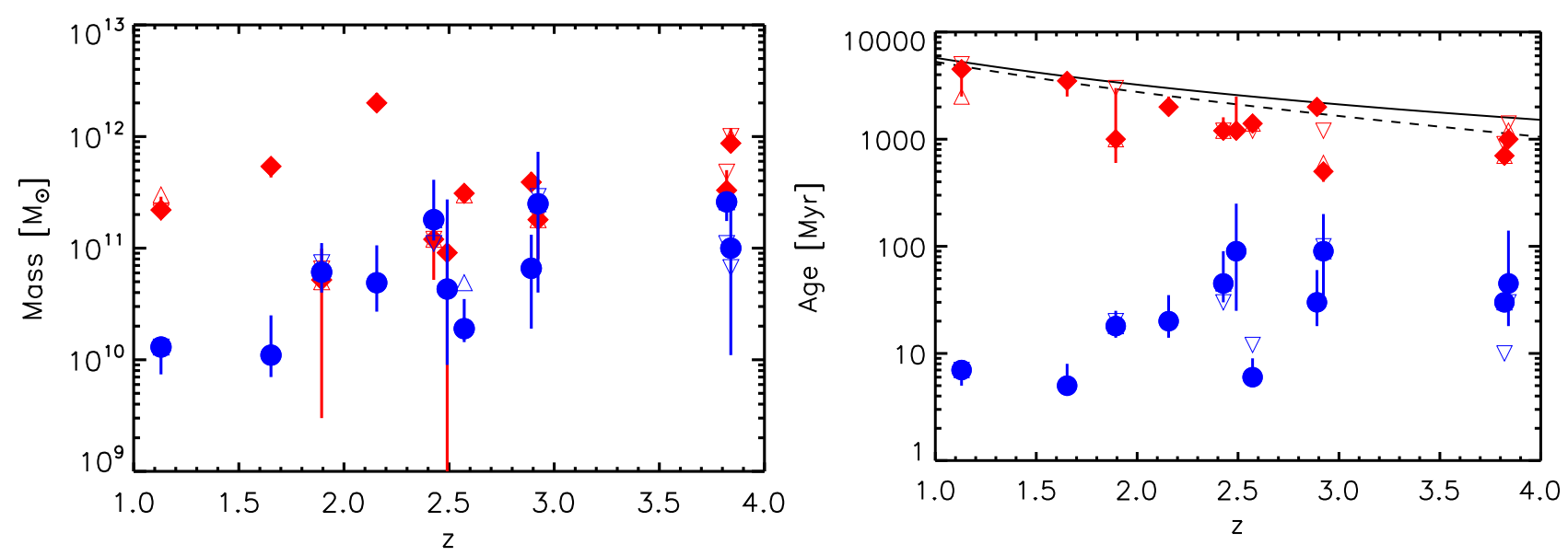

Fig. 3. Two stellar populations, young and evolved, shown as blue circles and red diamonds, respectively. The vertical lines indicate the $68 \%$ confidence intervals from the fit. When polarisation data are available, we report the fit with lowest and highest contribution from scattered AGN light as the downward and upward empty triangles respectively. For clarity, we do not add the $68 \%$ confidence intervals for the polarisationsubtracted fits. Left: masses of the two stellar populations versus redshift. Right: ages of the two stellar populations where the black line shows the oldest universe age as a function of redshift assuming the standard cosmological concordance model (Sect. 1). The dashed line represents the age of a galaxy for $z_{\text {form }}=10$. We note that the $68 \%$ confidence interval can be smaller than point size and polarisation-subtracted fits are mainly contained in the $68 \%$ confidence intervals when scattered AGN light is ignored.

\subsection{Intense and massive starburst}

Figure 3 and Table 4 show two remarkable features of the young component required to reproduce the strong Herschel and submm emission in our sample. Firstly, the starburst is extremely massive, $M_{\text {stel }}>10^{10} M_{\odot}$. In each case, it represents a significant fraction of the mass of the system (5\% to 50\%). These high-mass fractions indicate that even if the bulk of the stars has already been formed at high-redshift (Sect. 4.1), the radio galaxies in our sample still experience vigorous star formation ${ }^{3}$. Secondly, Fig. 3 also shows that this starburst spans a wide range in age, $5 \mathrm{Myr}<t_{\mathrm{SB}}<100 \mathrm{Myr}$, indicating that star formation is ongoing or in the aftermath of a violent event.

It is also interesting to note that all but one (MRC 2104242 ) of the galaxies have a column density factor $K=10$. This indicates that starbursts associated with radio galaxies have an on average ten times higher column density than the Milky Way ${ }^{4}$. This indicates that high-redshift radio galaxies, or at least the most IR-luminous radio galaxies, favour higher covering dust fractions, indicating a high obscuration, similar to SMGs in the same redshift range (e.g., Chen et al. 2015).

About half of the solutions favour a high initial metallicity (see Table 4). This could be expected for a starburst forming from IGM gas, which is expected to be metal-rich at highredshift (e.g., Pieri et al. 2014). Interestingly, the other half tends to have a low (or null) initial metallicity, indicating a more pristine gas source. The evolution of the starburst implies ISM enrichment when SN explode and therefore the observed metallicity will be higher than the one reported here (initial metallicity of the gas). Given that we used only broad-band photometry, the derived initial metallicities should be treated with a grain of caution, even though they do provide some clues about the overall metallicity of the initial gas of the starburst (null, sub-solar, or super-solar). The broad trend is well shown in Figs. D.1-D.10, where the best-fit solutions are shown not to be located in deep

\footnotetext{
3 We note that our sample selection requires bright IR emission $(\geq 4$ Herschel detections), implying the sample is likely biased towards strong star-forming systems.

$4 N_{\mathrm{HI}} \sim 6.8 \times 10^{21}$ atoms cm $\mathrm{cm}^{-2}$ is the standard galactic column density in PÉGASE.3.
}

and localised minima. Moderately high-resolution spectroscopic observations are necessary to further explore this part of the parameter space because the emission lines are the result of the ISM photoionisation from both the young stars and the AGN.

We make a final note about the star formation rate related to the starburst component. By definition (in the model), all the star formation is taking place in a single time step (we discuss the duration of SB in Sect. 5). When the fit converges to an age $>1 \mathrm{Myr}$, the related star formation is therefore null. Moreover, the absence of infrared imaging at arcsec resolution and the uncertainties about the duration of the burst make deriving a star formation rate from our fitting difficult. We therefore used simple physical assumptions and empirical relations to estimate star formation rates from the starburst component (see Sect. 5.2).

\subsection{Bolometrically luminous $A G N$ with an opaque torus}

Our SED fitting allows us to derive some of the AGN and torus properties of HzRGs, such as the size, opacity and inclination. With Eq. (1) we calculated the corresponding intrinsic luminosity of the AGN (Table 4, last column). All our AGN are luminous, with $L_{\mathrm{AGN}}^{\mathrm{int}} \gtrsim 10^{12} L_{\odot}$, most with $L_{\mathrm{AGN}}^{\mathrm{int}} \approx 10^{13-14} L_{\odot}$. Unfortunately, the small span of properties of our sample (in terms of luminosity or mass), usually only covering one order of magnitude, does not allow us to identify any correlation between the AGN and host properties. Interestingly, these values are similar to optically luminous quasars observed at similar redshift (e.g., Kollmeier et al. 2006). This is consistent with radio galaxies experiencing a strong radiation-dominated accretion phase with copious accretion onto the central supermassive black hole. In Figs. 2 and D.1-D.10, we show that the relative contribution at $20 \mu \mathrm{m}$ and therefore the AGN luminosity is well constrained.

Except for 3C 368, the tori in our sample appear opaque, with $\tau_{9.7 \mu \mathrm{m}} \geq 1.0$. This opacity corresponds to $A_{V}>18$ using the Draine (2003) conversion. The obscuration is also related to the inclination. The inclination represents the amount of dust on the line of sight. Most of our best fits converge to solutions with $i>40^{\circ}$, indicating Type 2 AGN (edge-on view), as expected for radio galaxies. The one exception is $3 \mathrm{C} 368$, the source with the lowest redshift in our sample, with $i=40^{\circ}$. The best solutions 
Table 4. Summary of the fitting results.

\begin{tabular}{lccccc}
\hline \hline \multirow{2}{*}{ Name } & \multicolumn{5}{c}{ Evolved component } \\
\cline { 2 - 6 } & $\begin{array}{c}\text { Hubble } \\
\text { type }\end{array}$ & $\begin{array}{c}\text { Mass } \\
\log _{10}\left[M_{\odot}\right]\end{array}$ & $\begin{array}{c}\text { Age } \\
{[\mathrm{Myr}]}\end{array}$ & $Z *$ & $L^{\text {tot }}$ \\
\hline 3C 368 & Sa & $11.3_{-0.0}^{+0.1}$ & $4500_{-2000}^{+500}$ & 0.0112 & $11.6_{-1.3}^{+0.2}$ \\
3C 470 & Sa & $11.7_{-0.1}^{+0.1}$ & $3500_{-1000}^{+0}$ & 0.0092 & $12.0_{-0.7}^{+0.3}$ \\
MRC 0324 & E2 & $10.7_{-1.2}^{+0.3}$ & $1000_{-400}^{+2000}$ & 0.0264 & $11.8_{-0.0}^{+0.0}$ \\
PKS 1138 & S0 & $12.3_{-0.0}^{+0.1}$ & $2000_{-200}^{+500}$ & 0.0142 & $12.7_{-1.0}^{+0.2}$ \\
MRC 0406 & E2 & $11.1_{-0.4}^{+0.1}$ & $1200_{-0}^{+400}$ & 0.0259 & $11.8_{-0.3}^{+0.3}$ \\
MRC 2104 & E2 & $11.0_{0.3}^{+0.3}$ & $1200_{-200}^{+1300}$ & 0.0259 & $11.7_{0.0}^{+0.0}$ \\
USS 0828 & E2 & $11.5_{0.0}^{+0.0}$ & $1400_{-0}^{+0}$ & 0.0257 & $12.2_{-0.0}^{+0.3}$ \\
4C 2858 & S0 & $11.6_{-0.0}^{+0.1}$ & $2000_{-200}^{+0}$ & 0.0142 & $12.0_{-1.1}^{+0.2}$ \\
USS 0943 & E & $11.3_{-0.4}^{+0.1}$ & $500_{-100}^{+100}$ & 0.0114 & $12.1_{-0.2}^{+0.2}$ \\
4C 4117 & S0 & $11.5_{-0.1}^{+0.2}$ & $700_{-100}^{+300}$ & 0.0057 & $12.3_{-0.6}^{+0.2}$ \\
TNJ 2007 & E & $11.9_{-0.1}^{+0.1}$ & $1000_{-100}^{+200}$ & 0.0148 & $12.4_{-0.7}^{+0.2}$ \\
\hline
\end{tabular}

\begin{tabular}{|c|c|c|c|c|c|}
\hline \multirow[b]{2}{*}{ Name } & \multicolumn{5}{|c|}{ SB component* } \\
\hline & $\begin{array}{c}\text { Mass } \\
\log _{10}\left[M_{\odot}\right]\end{array}$ & $\begin{array}{c}\text { Age } \\
{[\mathrm{Myr}]}\end{array}$ & $Z_{\text {init }}$ & $K$ & $\begin{array}{c}L^{\text {tot }} \\
\log _{10}\left[L_{\odot}\right]\end{array}$ \\
\hline 3C 368 & $10.1_{-0.2}^{+0.1}$ & $7_{-2}^{+1}$ & 0.01 & 10 & $12.7_{-0.4}^{+0.3}$ \\
\hline $3 C 470$ & $10.0_{-0.2}^{+0.4}$ & $5_{-1}^{+3}$ & 0.01 & 10 & $12.9_{-0.5}^{+0.1}$ \\
\hline MRC 0324 & $10.8_{-0.2}^{+0.3}$ & $18_{-4}^{+7}$ & 0.01 & 10 & $12.9_{-0.5}^{+0.1}$ \\
\hline PKS 1138 & $10.7_{-0.3}^{+0.3}$ & $20_{-6}^{+15}$ & 0.0005 & 10 & $13.2_{-0.4}^{+0.1}$ \\
\hline MRC 0406 & $11.3_{-0.2}^{+0.4}$ & $45_{-15}^{+45}$ & 0.0 & 10 & $13.1_{-0.4}^{+0.1}$ \\
\hline MRC 2104 & $10.6_{-0.7}^{+0.8}$ & $90_{-65}^{+160}$ & 0.0 & 1 & $12.9_{-0.1}^{+0.4}$ \\
\hline USS 0828 & $10.3_{-0.1}^{+0.3}$ & $6_{-1}^{+3}$ & 0.01 & 10 & $13.2_{-0.6}^{+0.1}$ \\
\hline 4C 2858 & $10.8_{-0.5}^{+0.3}$ & $30_{-12}^{+30}$ & 0.005 & 10 & $13.3_{-0.1}^{+0.0}$ \\
\hline USS 0943 & $11.4_{-0.8}^{+0.5}$ & $90_{-60}^{+110}$ & 0.01 & 10 & $13.0_{-0.1}^{+0.1}$ \\
\hline $4 C 4117$ & $11.4_{-0.2}^{+0.0}$ & $30_{-5}^{+5}$ & 0.001 & 10 & $13.4_{-0.5}^{+0.3}$ \\
\hline \multirow[t]{2}{*}{ TNJ 2007} & $11.0_{-1.0}^{+0.4}$ & $45_{-27}^{+95}$ & 0.001 & 10 & $13.0_{-0.1}^{+0.2}$ \\
\hline & \multicolumn{5}{|c|}{ AGN component } \\
\hline Name & $Y$ & $\tau_{9.7 \mu \mathrm{m}}$ & $\begin{array}{c}i \\
\text { [degree }\end{array}$ & & $\begin{array}{c}L^{\text {tot }} \\
\log _{10}\left[L_{\odot}\right]\end{array}$ \\
\hline 3C 368 & $150_{-0}^{+0}$ & $0.1_{-0.0}^{+0.0}$ & $40_{-0}^{+0}$ & & $11.7_{-0.4}^{+0.3}$ \\
\hline $3 C 470$ & $60_{-0}^{+90}$ & $10.0_{-0.0}^{+0.0}$ & $50_{-0}^{+10}$ & & $13.2_{-0.4}^{+0.5}$ \\
\hline MRC 0324 & $60_{-0}^{+90}$ & $1.0_{-0.9}^{+0.0}$ & $90_{-10}^{+0}$ & & $12.3_{-0.2}^{+0.4}$ \\
\hline PKS 1138 & $10_{-0}^{+0}$ & $1.0_{-0.0}^{+0.0}$ & $50_{-0}^{+10}$ & & $13.5_{-0.3}^{+0.5}$ \\
\hline MRC 0406 & $10_{-0}^{+0}$ & $10.0_{-0.0}^{+0.0}$ & $50_{-0}^{+20}$ & & $13.3_{-0.4}^{+0.5}$ \\
\hline MRC 2104 & $150_{-90}^{+0}$ & $10.0_{-0.0}^{+0.0}$ & $80_{-30}^{+10}$ & & $13.7_{-0.1}^{+0.9}$ \\
\hline USS 0828 & $10_{-0}^{+0}$ & $1.0_{-0.0}^{+0.0}$ & $70_{-0}^{+10}$ & & $13.5_{-0.6}^{+0.5}$ \\
\hline 4C 2858 & $\begin{array}{l}60_{-0}^{+0} \\
\text { - }\end{array}$ & $10.0_{-0.0}^{+0.0}$ & $90_{-0}^{+0}$ & & $14.3_{-0.1}^{+0.0}$ \\
\hline USS 0943 & $150_{-140}^{+0}$ & $10.0_{-0.0}^{+0.0}$ & $70_{-20}^{+10}$ & & $13.4_{-0.0}^{+0.6}$ \\
\hline 4C 4117 & $10_{-0}^{+140}$ & $10.0_{-0.0}^{+0.0}$ & $80_{-10}^{+10}$ & & $13.9_{-0.5}^{+0.3}$ \\
\hline TNJ 2007 & $10_{-0}^{+0}$ & $\begin{array}{l}1.0_{-0.0}^{+0.0} \\
0^{2}\end{array}$ & $50_{-0}^{+20}$ & & $13.0_{-0.1}^{+0.8}$ \\
\hline
\end{tabular}

Notes. The $\chi^{2}$ is given in the last column of Table 3 . The table is divided in three parts corresponding to each fitted component. For the evolved component: $Z_{*}$ refers to the average metallicity lock in stars for the given age. For the SB component: $Z_{\text {init }}$ refers to the initial metallicity of the gas, $K$ is the factor relating to the column density. For the AGN component: $Y$ refers to the size ratio between the outer and inner radius of the torus, $\tau_{9.7 \mu \mathrm{m}}$ the opacity at $9.7 \mu \mathrm{m}$, and $i$ to the inclination with respect to the observer. Asymmetric uncertainties of each parameter are provided as explained in Sect. 3.3. We note that zero values of the uncertainties are due to the lack of coverage in the parameter space and are contained in the $68 \%$ uncertainties, refer to Figs. D.1-D.10.

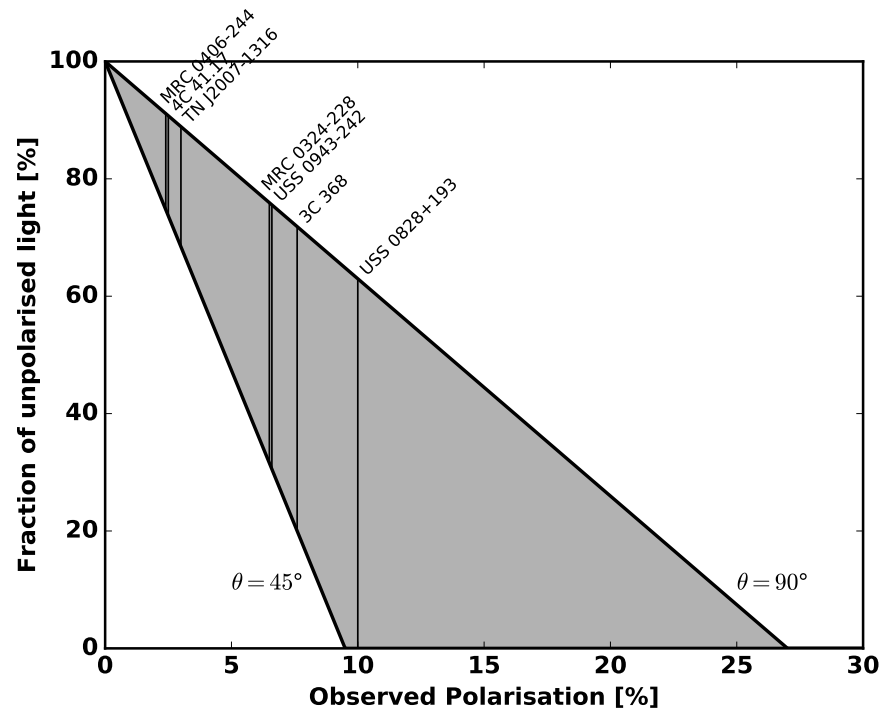

Fig. 4. Highest and lowest polarisation diagram at $1500 \AA$ A. The grey shaded area represents the allowed fraction of unpolarised light depending on the inclination (see text). Polarisation measurements of our sources are reported as vertical lines without the uncertainties for visibility (i.e. the uncertainties increase the allowed range of unpolarised light accordingly).

vary from $Y=10$ to $Y=150$ for the size. As we calculated the intrinsic luminosity, we can estimate the physical size of the torus (see Eq. (1) of Fritz et al. 2006). Taking the median of our sample, $L_{\mathrm{AGN}}^{\text {int }} \sim 2.5 \times 10^{13} L_{\odot}$ translates into $R_{\min } \sim 4.1 \mathrm{pc}$ and $R_{\max } \sim 41 \mathrm{pc}, \sim 246 \mathrm{pc}$, and $\sim 616 \mathrm{pc}$ for $Y=10,60$, and 150 , respectively. We note the difficulties in constraining the torus properties are expected because even with dedicated samples in the nearby Universe, some parameters are only loosely constrained (e.g., Asensio Ramos \& Ramos Almeida 2009). Interestingly, even after chosing the most relevant parameters for the AGN torus, the properties of the torus vary from source to source, with no clear trend on the entire sample (see Figs. 2 and D.1-D.10).

\subsection{Moderate effect of AGN-scattered light in broad-band SED fitting}

The AGN emission is partly absorbed by the dusty torus or escapes along the open part of the torus. Escaping photons can be scattered by dust present in the ionisation cone toward the observer, and thereby contaminate the overall UV and optical light. This contribution especially affects type 2 AGN (seen edge-on), and can be strong in the UV and optical rest-frame (up to $100 \%$ di Serego Alighieri et al. 1996; Cimatti et al. 1998; Vernet et al. 2001). It is important to quantify this effect in our SED fitting. We used the technique from Vernet et al. (2001) to estimate the range of possible contributions of the optical AGN scattered light, as illustrated in Fig. 4. We stress that this range relies on several assumptions regarding the geometry and inclination of the obscuring dust distribution, which are consistent with the adopted torus models $\left(\theta=40^{\circ}\right.$, inclination $\left.i\right)$.

One fundamental property assumed here is the grey behaviour of polarisation, that is, the fact that polarisation from dust scattering is approximately independent of wavelength (Vernet et al. 2001). This allows us to estimate the range of AGN contribution in the UV and optical domain from a single polarisation measurement at $1500 \AA$. When a polarisation measurement was available, we therefore considered the following three cases: (i) without polarisation; (ii) with lowest possible 
AGN contamination; and (iii) with the highest possible AGN contamination. We applied the following correction to the UV and optical photometry before the fitting: we scaled the $1500 \AA$ contribution from Fig. 4 and subtracted the corresponding fraction from our broad-band photometry ${ }^{5}$ with a typical UV and optical continuum quasar spectrum from Cristiani \& Vio (1990). The latter represents a typical UV and optical SED of a type 1 AGN (i.e. without obscuration). We recall that these are the boundary cases and that the true contamination is located between these two extremes.

The most polarised source of our sample, USS $0828+193$, with $P=10.0 \pm 2.0 \%$ is predicted to have a contribution from unpolarised light of between $0 \%$ and $\sim 65 \%$. The remaining AGN contribution is thus between $\sim 35$ and $100 \%$ (illustrated in Fig. 5). Subtracting the lowest (35\%) AGN contribution has almost no effect on the fit $\left(\chi^{2}=35.7\right.$ or $\chi^{2}=37.1$, respectively). This suggests either that (i) the addition of this component is not particularly justified, or that (ii) the true contamination is not close to the lowest $35 \%$ contribution of the AGN. As the highest possible AGN contribution for this source is $100 \%$ in the UV, this would leave no room for any stellar contribution. Such an exceptional case (only one in our sample) cannot be properly taken into account in our fitting procedure. We therefore consider the lowest possible AGN contribution as an upper limit on the effect on USS $0828+193$. For this lowest contribution, the effect on the fitting is limited to a factor of $\sim 2$ for the mass and $\sim 2$ for the age of the stellar components (see Fig. 3). For the remaining six sources with polarisation measurements, the difference induced by polarisation is typically contained in our $68 \%$ confidence intervals (see empty symbols in Fig. 3). The effect on the other parameters is presented in the insets in the SEDs in Figs. 2 and D.1-D.10 (blue and red points for the lowest and highest contamination, respectively).

We conclude that the effect of polarisation for our broadband SED fitting is weaker than our global uncertainties on each parameter. Polarisation therefore has a negligible effect on our main conclusion, and we ignore this in the remainder of this paper.

\section{Discussion}

Our fitting procedure enables us to carefully disentangle the three main spectral components of our sources: an evolved stellar component, an SB component and an AGN. We focus on the new information provided by the Herschel data in the farinfrared (FIR), allowing us to disentangle the SB from the AGN contribution. We find that the two stellar components are massive $\left(>10^{10} M_{\odot}\right)$ and that both the AGN and the SB are very luminous $\left(>10^{10} L_{\odot}\right)$. The properties of the evolved stellar population confirm a very high formation redshift of the galaxy host, leading to local massive early-type galaxies as previously proposed in literature (e.g. Lilly \& Longair 1984). Interestingly, our sources have similar properties as quasars and SMGs in the same redshift range (e.g., Alexander et al. 2005; Wang et al. 2011; Leipski et al. 2014, see Sect. 4). We discuss the limitations of our modelling in a first step and then explore the properties of star formation making use of physical arguments and empirical laws from SED fitting. Finally, we classify and discuss

\footnotetext{
5 We are interested only in the continuum emission, therefore we removed the strong emission lines from the templates by linearly interpolating the continuum below the line. In addition, line polarisation is typically close to null because the physical processes involved in line emission are different from the continuum.
}
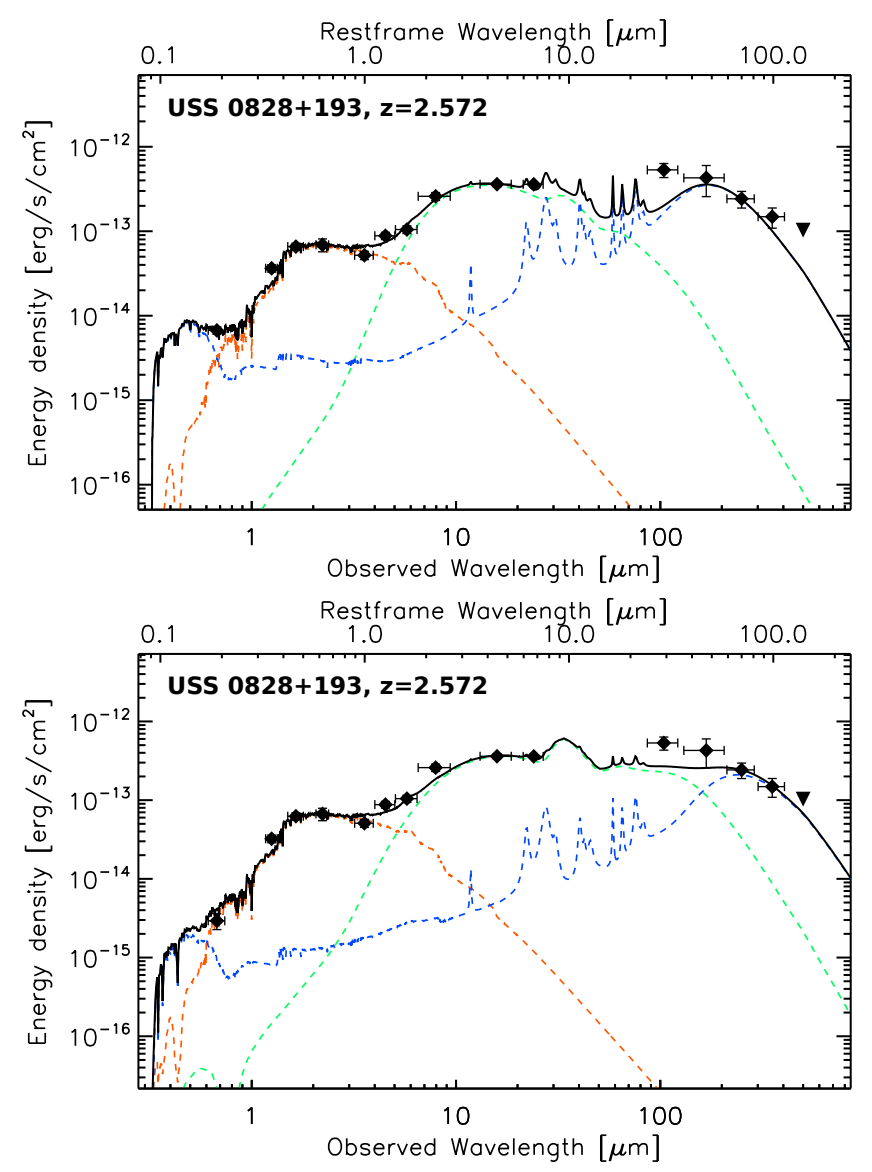

Fig. 5. SEDs of USS $0828+193$ showing the impact of the polarisation on our fitting. The colour coding is the same than Fig. 2. The top panel is without polarisation, while the bottom panel is the minimal subtraction case. The maximal case of a $100 \%$ subtraction is not reported (see text).

the morphology of our sources making use of a combination of multi-wavelength data.

\subsection{Modelling uncertainties, limitations, and multi-wavelength data set}

The self-consistency in our models is a key point of our modelling: for instance, the computed FIR emission is dependent on the dust properties defined by the evolutionary scenario and produced from the stellar population evolution (ISM enrichment, see Sect. 3.1.1). We discuss here the limitations and degeneracies within our libraries and from our observations on the fitting procedure. We refer to Sect. 3 and Appendix A for the discussion of the model assumptions and systematics introduced by our choice of code and libraries.

The PÉGASE. 3 model only explores a limited number of star formation histories. For the evolved component, the star formation history is set by the Hubble type, although the data presented here are unable to prefer a single type within the confidence intervals (Sect. 3.3). However, the effect on the age and mass of stellar populations is limited, within a factor of 2 (see RV13 for better details). For the starburst, the star formation is considered instantaneous (in one time step). Forming $\sim 10^{10} M_{\odot}$ in such a short time appears highly unlikely. Nevertheless, we note that only a short burst can reproduce the strong submm emission in our sample. We stress that star formation is not necessarily 
spatially concentrated, but is most probably temporally connected. Therefore, the SB component may be interpreted as a collection of smaller clumps, formed in a short period. In addition, RV13 have discussed the effect of this $\delta$ function, showing that the age from the fitting is measured within a factor of $\sim 2$ for constant star formation over a longer period of time.

The Fritz et al. (2006) AGN model is one of several models available in the literature. Direct comparisons between different models are difficult because of the variation in the number of free parameters and because of the assumptions on the torus properties itself (smooth, clumpy, bi-phased). More investigations are reserved for further papers, taking different AGN models into account (e.g. Podigachoski et al., in prep.). We therefore do not further discuss the implications of the smooth torus model on our analysis, but we warn that the results provided here are dependent on this choice. We note that part of our best fit has difficulties to reproduce the $30 \mu \mathrm{m}$ restframe data (e.g. PKS 1138-262 and USS 0828+193). The most likely explanation is the lack of exploration in the parameter space, especially on the size parameter. A larger torus, having dust at larger radii, would better reproduce this cold emission.

Finally, observations themselves can be affected by systematics. This is particularly true for our set of multi-wavelength observations that were obtained with a range of instruments and telescopes, each presenting their own calibration uncertainties, especially at moderate signal-to-noise ratio. The FIR and submm domain are particularly challenging observations and the photometry estimation method can have a significant effect on the final measurement, especially in the $<5 \sigma$ range (by a factor of $\sim 20 \%$; Popesso et al. 2012). A good illustration is the $160 \mu \mathrm{m}$ photometry in 4C 28.58 (Fig. D.8), where the $160 \mu \mathrm{m}$ flux might be expected to be higher. Similarly, sky-subtraction strategy (onoff versus raster) may affect the $850 \mu \mathrm{m}$ photometry, possibly explaining the differences in the cold dust part of the SED. These uncertainties are mitigated by the good sampling of our SEDs, however.

\subsection{Star formation properties from the SED fitting}

In the previous sections we estimated several crucial properties related to the star formation in our sample, such as the age, average column density, mass and, to some extent, initial metallicity. Even if the star formation most likely occurs simultaneously in different clumps, we treat this component as a single idealised starburst (completely localised and isolated). This provided us with information useful for comparing our sample to other galaxies. Assuming the starburst has a projected circular geometry (at constant density), we estimate the size of the starburst region as follows:

$r_{\mathrm{SB}} \propto\left(\frac{M_{\mathrm{SB}}}{\pi K N_{\mathrm{HI}}}\right)^{\frac{1}{2}}$,

where $r_{\mathrm{SB}}$ is the projected radius, $M_{\mathrm{SB}}$ is the mass of the starburst, $K$ is the column density factor and $N_{\mathrm{HI}}$ the column density (here $N_{\mathrm{HI}}=6.8 \times 10^{21}$ atoms $\mathrm{cm}^{-2}$ ). These values are reported in the third column of Table 5. With an estimated size, we can define a typical velocity dispersion assuming equilibrium:

$\sigma_{\mathrm{SB}} \sim \sqrt{\frac{G M_{\mathrm{SB}}}{r_{\mathrm{SB}}}}$,

where $\sigma_{\mathrm{SB}}$ is the velocity dispersion of the gas, and $G$ is the gravitational constant. We recall that when the system is collapsing, this value is an upper limit, while for an expanding system, this value becomes a lower limit. Assuming the relation
$V_{\text {circ }}=\sigma / 0.6$ (e.g., Rix et al. 1997), we calculate the dynamical time as $t_{\mathrm{dyn}}=2 r_{\mathrm{SB}} / \sigma_{\mathrm{SB}}$.

The projected star formation per surface unit is a powerful proxy to estimate the physical conditions of the starburst (e.g., Lehnert \& Heckman 1996). Based on the physical sizes and timescales derived above, we calculate the star formation density, $\Sigma_{\mathrm{SFR}}$, with the following formula $\Sigma_{\mathrm{SFR}}=\operatorname{SFR} /\left(2 \pi \mathrm{r}_{\mathrm{SB}}^{2}\right)$ with $S F R=M_{\mathrm{SB}} / t_{\mathrm{dyn}}$.

It is interesting to note that the overall properties from these empirical calculations indicate (i) a high efficiency of forming stars with an sSFR $>20 \mathrm{Gyr}^{-1}$ in all cases, consistent with measurements from Drouart et al. (2014); (ii) a dense star-forming region $\Sigma_{\mathrm{SFR}}>5 M_{\odot} \mathrm{yr}^{-1} \mathrm{kpc}^{-2}$, similar to the local starburst (e.g. Bigiel et al. 2008) and largely exceeding the limit for starburst driven winds (e.g. Lehnert \& Heckman 1996); and (iii) half (5/11 sources) of our sample presents an SB mass of the same order as the host component (Table 5), strongly suggesting merging activity. These estimates have important implications in terms of star formation properties because it suggests that the star formation is very efficient in very confined regions. However, the large projected radius ( $>1 \mathrm{kpc})$, suggests that the star formation arises in several locations within the same galaxy.

\subsection{Localisation of the star formation from the images}

The limited spatial resolution of Herschel makes it difficult to answer the key questions of the location of star formation and of the process that leads to the observed star formation. Complementary to the spectral information, spatial information can be used to understand the localisation of star formation and the overall evolutionary status of the radio galaxies in our sample. We made use of the high-resolution HST and radio imaging, along with the moderate resolution of the NIR data, to investigate the relative location of the emission at different wavelengths ${ }^{6}$. We visually classified our sources into three non-exclusive categories as follows (and report their likelihood in Table 5 as none, low, medium, and high):

1. "ex situ star formation", where star formation occurs outside the galaxy, at $>5 \mathrm{kpc}$ of the radio galaxy (late-stage mergers and star-forming companions fall into this category);

2. "in situ star formation", which refers to star formation that occurs within the radio galaxy, at $<5 \mathrm{kpc}$ (rotating structure and nuclear star formation fall into this category);

3. "AGN-driven star formation" corresponding to star formation linked to AGN activity, independently of spatial scale (both jet-induced and shock-induced star formation fall into this category).

The limit of $5 \mathrm{kpc}$ is driven by the resolution obtained in the optical and $\mathrm{NIR}^{7}$ domains and the typical size of a massive galaxy at $z>1$ (Fan et al. 2010).

\subsubsection{Ex situ star formation}

Ex situ star formation can be divided into two subcategories, a massive star-forming companion or a multiple system of less massive sub-units, which can be interpreted as a signature of

\footnotetext{
6 The HST data are downloaded from the HLA. The Herschel data are unresolved when compared to optical or NIR images and are not reported for simplicity (but available in Drouart et al. 2014).

7 We recall that at $z>1,1^{\prime \prime}$ corresponds roughly to $8 \mathrm{kpc}$, assuming the standard cosmological model.
} 
G. Drouart et al.: Disentangling SF and AGN in luminous HzRGs

Table 5. Physical properties of the star formation, assuming empirical relations and simple physical assumptions for an isolated starburst (Cols. 2-6, see also Sect. 5.2).

\begin{tabular}{|c|c|c|c|c|c|c|c|c|}
\hline \multirow[b]{2}{*}{ Radio galaxy } & \multirow[b]{2}{*}{$\begin{array}{c}\frac{M_{\mathrm{SB}}}{M_{\mathrm{tot}(\mathrm{SB}+\text { evol. }}} \\
{[\%]} \\
\end{array}$} & \multicolumn{4}{|c|}{ Empirical properties from SED } & \multicolumn{3}{|c|}{ Visual association of from images } \\
\hline & & $\begin{array}{c}r_{\mathrm{SB}} \\
{[\mathrm{kpc}]}\end{array}$ & $\begin{array}{c}t_{\mathrm{dyn}} \\
{[\mathrm{Myr}]}\end{array}$ & $\begin{array}{c}\mathrm{s} S F R \\
{\left[\mathrm{Gyr}^{-1}\right]}\end{array}$ & $\begin{array}{c}\Sigma_{\mathrm{SFR}} \\
{\left[M_{\odot} \mathrm{yr}^{-1} \mathrm{kpc}^{-2}\right]}\end{array}$ & ex situ (>5 kpc) & $\begin{array}{l}\text { in situ }(<5 \mathrm{kpc}) \\
\text { likelihood }\end{array}$ & AGN-driven \\
\hline $3 \mathrm{C} 470$ & 2 & 2.4 & 21 & 46 & 12.8 & medium? & medium? & none \\
\hline MRC 0324-228 & 56 & 5.4 & 31 & 31 & 8.5 & $\ldots$ & $\ldots$ & $\ldots$ \\
\hline PKS 1138-262 & 2 & 6.8 & 35 & 27 & 7.6 & high & low & none \\
\hline USS $0828+193$ & 6 & 3.4 & 25 & 39 & 10.7 & none & high & medium? \\
\hline 4C 28.58 & 14 & 6.1 & 33 & 29 & 8.1 & medium? & medium? & medium? \\
\hline USS $0943-242$ & 56 & 12.1 & 47 & 20 & 5.7 & high & low & none \\
\hline 4C 41.17 (all) & 44 & 12.1 & 47 & 20 & 5.7 & high & low & medium \\
\hline TN J2007-1316 & 11 & 6.1 & 33 & 29 & 8.1 & high & $\ldots$ & $\ldots$ \\
\hline
\end{tabular}

Notes. All calculated values only take into account the SB component (see Sect. 5.2 for details). None of the indicated properties are fully constrained (except for 4C 41.17 (compact) where we constrain the size; see Sect. 5.3.3 for details). Columns 7-9 refer to the likelihood from visual inspection on images described in Sect. 5.3. Question marks refer to ambiguous case (see respective figures). (...) indicates a lack of data to assess any visual association.

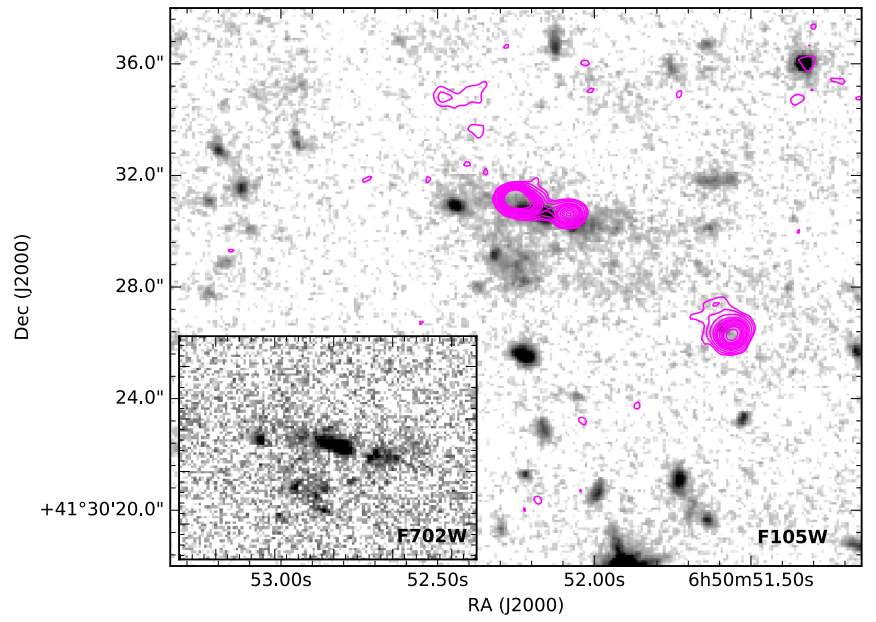

Fig. 6. Composite view of $4 \mathrm{C} 41.17(z=3.792)$. The main frame is a $1.05 \mu \mathrm{m}$ HST image while the inset corresponds to the $R$ band. The magenta contours show the $4.8 \mathrm{GHz}$ data overlaid at $3,5,10,20,30$, $50,75,100,125,150$, and $200 \sigma$, with an rms $=25 \mu \mathrm{Jy}$.

a major merger or multiple minor mergers, respectively. Based on our visual classification (Col. 7 in Table 5), seven of the eleven sources have a medium or high likelihood of ex situ star formation.

Among the "high" likelihood ex situ star forming sources, three sources have a SB mass fraction $>28 \%$ (4C 41.17 Fig. 6, USS 0943-242, Fig. 7 and MRC 2104-242, Fig. 8), strongly suggesting major-merger events ${ }^{8}$. The four remaining sources (3C 470 - Fig. 9, PKS 1138-262 - Fig. 10, 4C 28.58 and TN J2007-1316 - Fig. 11) present a mass fraction for the SB in the $2-14 \%$ range, favouring a minor-merger scenario or orbiting

\footnotetext{
8 Recently, USS 0943-242 was observed at submm wavelength at arcsec resolution, indicating that a significant fraction of star formation occurs outside the host galaxy (Gullberg et al. 2016), which confirms the ex situ star formation scenario.
}

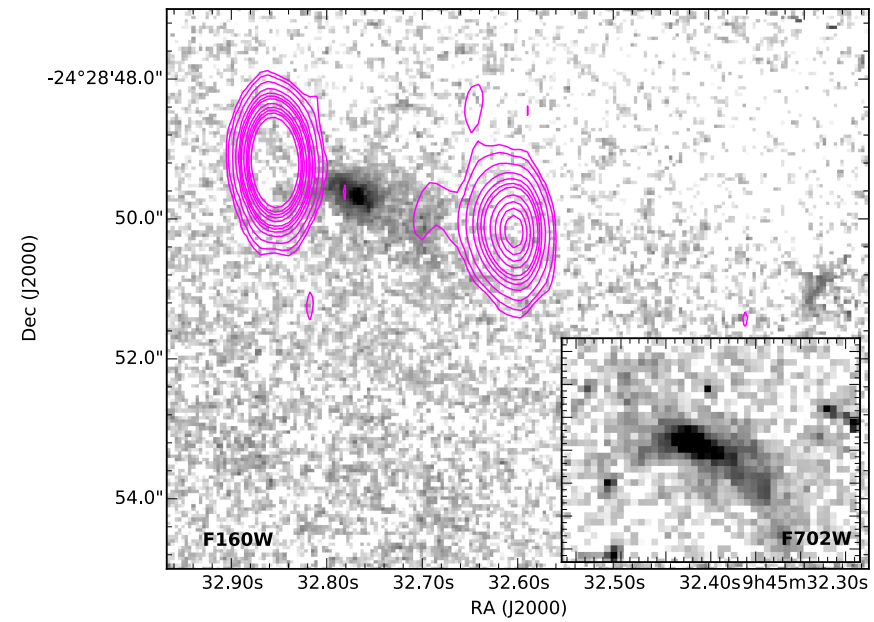

Fig. 7. Composite view of USS $0943+193(z=2.923)$. The main frame is a $1.6 \mu \mathrm{m}$ HST image while the inset corresponds to the $R$ band. The magenta contours are the $4.8 \mathrm{GHz}$ data overlaid at $3,5,10,20,30,50$, $75,100,125,150$, and $200 \sigma$, with an $\mathrm{rms}=50 \mu \mathrm{Jy}$.

companions. This is particularly clear in PKS 1138-262 where the HST images reveal many companions that are organised in a complex structure around the central massive galaxy that is located at the position of the radio core (Pentericci et al. 1998, see also Fig. 10). Almost all these companions are confirmed to be at the same redshift as PKS 1138-262 (Hatch et al. 2009; Doherty et al. 2010).

A note should be made on MRC 0324-228 (Fig. 12), where the SB mass fraction is very high $(56 \%)$, but where the lack of high-quality data prevents any clear association with star formation. The $K$ s-band image suggests that the star formation originates from a close companion or the radio galaxy itself.

When this classification is compared with the empirical properties of star formation, all seven sources but one (3C 470 Fig. 9) present a projected radius $r_{\mathrm{SB}}>4.8 \mathrm{kpc}$ (see Table 5) for the idealised starburst. This size is comparable to the size of 


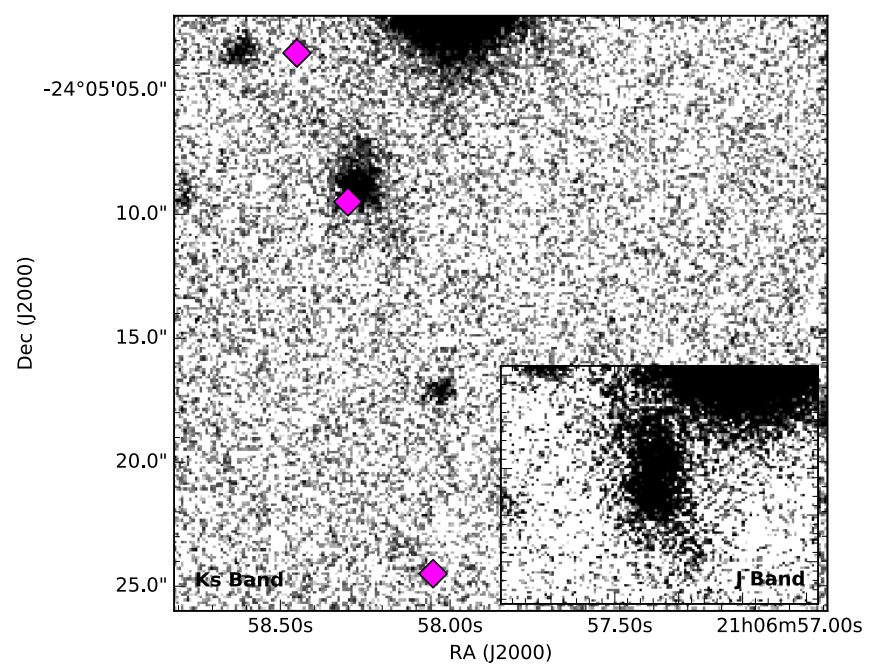

Fig. 8. Composite view of MRC 2104-242 $(z=2.491)$. The main frame is a $2.2 \mu \mathrm{m}$ image while the inset corresponds to the $I$ band. The magenta diamond shows the approximate position of the radio emission peak at $8.2 \mathrm{GHz}$ (Pentericci et al. 2001).

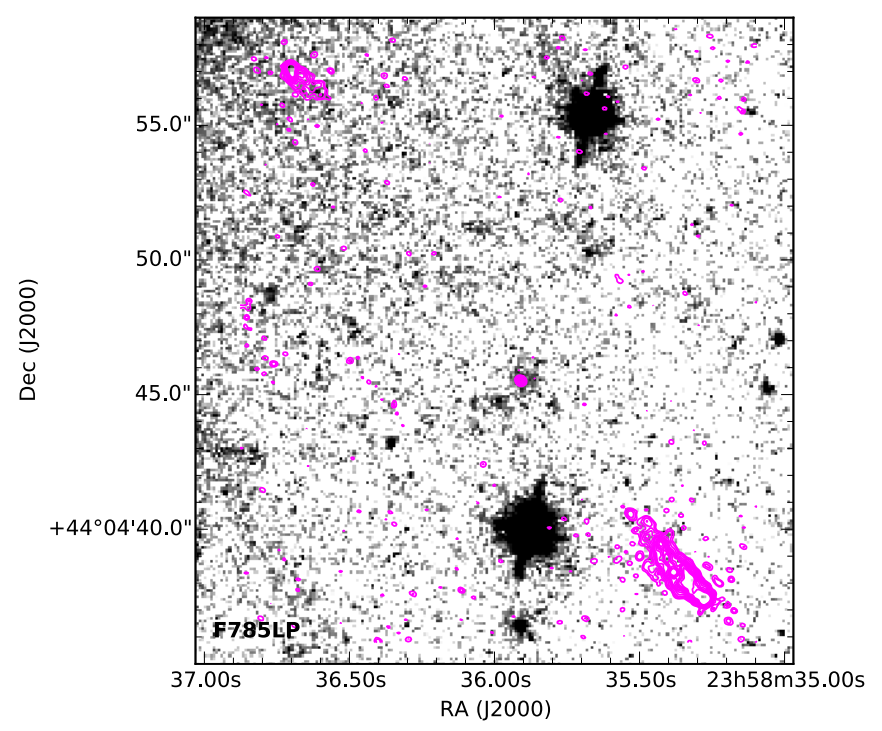

Fig. 9. Composite view of $3 \mathrm{C} 470(z=1.653)$. The main frame is a $z$ band HST image. The magenta contours show the $8.4 \mathrm{GHz}$ data overlaid at $3,5,10,20,30,50,75,100,125,150$, and $200 \sigma$, with an rms $=$ $25 \mu \mathrm{Jy}$.

a galaxy itself at these redshift, which additionally support the multiple galaxies and merging scenarios.

In our sample, $65 \%$ of our sources show evidence of merging activities (both major and minor). While the size of our sample is limited, this result agrees with those of other studies. Several galaxies from our parent powerful radio galaxy sample are known to be gas-rich major mergers (e.g., TXS J1809+7220, B3 $\mathbf{J} 2330+3927$, and MRC0152-209), but this is based on observations made outside the IR domain, on molecular gas mapping (De Breuck et al. 2005; Ivison et al. 2008, 2012; Emonts et al. 2014, 2015).

\subsubsection{In situ star formation}

In situ star formation refers to star formation that occurs in the radio galaxy host $(<5 \mathrm{kpc})$. Once again, two subcategories can

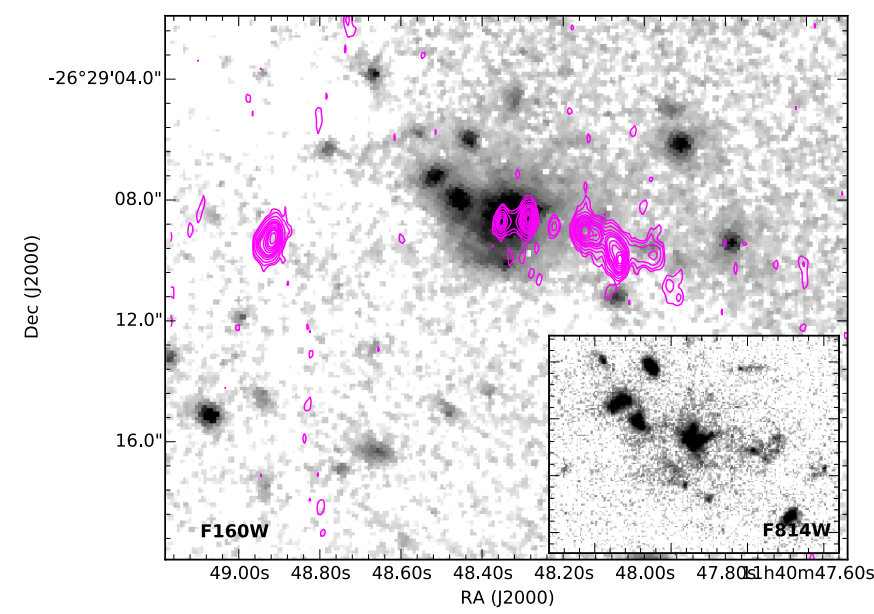

Fig. 10. Composite view of PKS 1138-262 $(z=2.43)$. The main frame is at $1.6 \mu \mathrm{m}$ HST image while the inset corresponds to the $I$ band. The magenta contours show the $8.4 \mathrm{GHz}$ data overlaid at $3,5,10,20,30,50$, $75,100,125,150$, and $200 \sigma$, with an rms $=30 \mu \mathrm{Jy}$. Note that most of the sources around the radio galaxy are at the same redshift (Hatch et al. 2011).

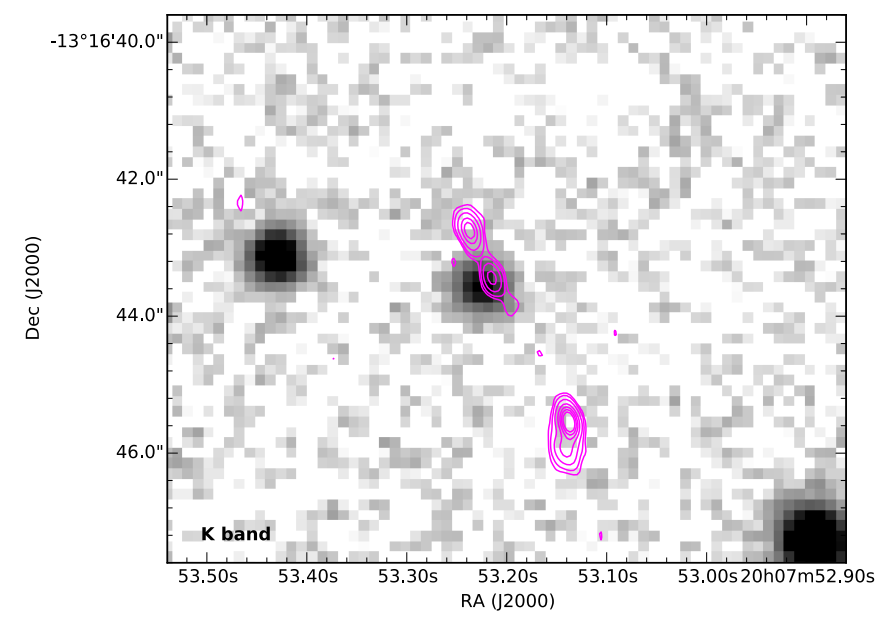

Fig. 11. $K$ s-band image of TN J2007-1316 $(z=3.840)$. The magenta contours are the $8.4 \mathrm{GHz}$ data overlaid at $3,5,10,20,30,50,75,100$, 125,150 , and $200 \sigma$, with an rms $=40 \mu \mathrm{Jy}$

be defined: a rotating star-forming structure from accreted gas, or a nuclear starburst embedded in the central part of the galaxy.

Three of our sources (3C 368 in Fig. 15, MRC 0406-244 in Fig. 13 and USS $0828+193$ in Fig. 14) show a high likelihood for in situ star formation (Table 5), representing 30\% of our sample (55\% when medium likelihood is included). MRC 0406-244 suggests an SB mass fraction of up to $61 \%$, while in 3C 368 and USS $0828+193$ the SB fraction is more moderate at $6 \%$ of the total mass. The case of MRC 0406-244 appears related to a rotating star-forming structure. Hatch et al. (2013) showed that the light profile is consistent with a disc, and the galaxy forming stars up to $1000 M_{\odot} \mathrm{yr}^{-1}$ (albeit with large uncertainties). Moreover, the system appears well isolated with no merger remnants seen in the HST images (Fig. 13). According to the SFR- $M_{*}$ relation at $z \sim 2$ (Rodighiero et al. 2011), a main-sequence galaxy in the $10^{11-12} M_{\odot}$ range will have a corresponding SFR in the 200-1000 $M_{\odot} \mathrm{yr}^{-1}$ range, which appears to be consistent with the specific case of MRC 0406-244. 


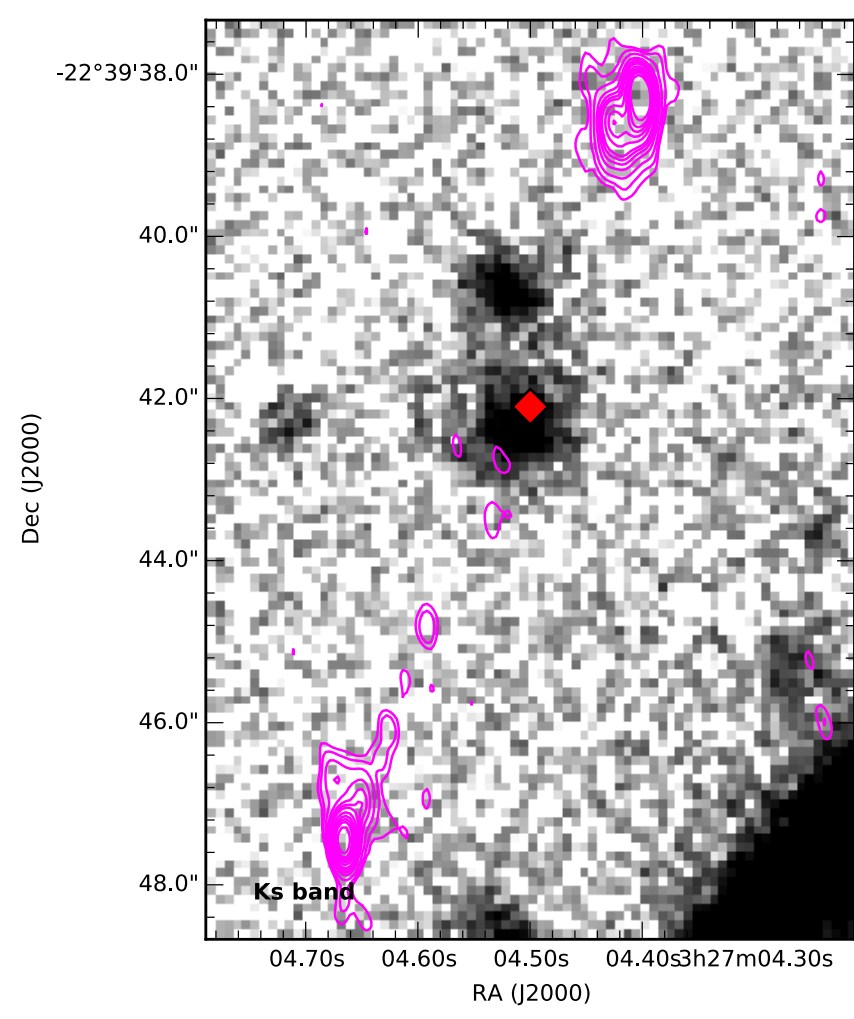

Fig. 12. Composite view of MRC 0324-228 $(z=1.874)$. The main frame is at $2.2 \mu \mathrm{m}$ image. The magenta contours are the $8.4 \mathrm{GHz}$ data overlaid at $3,5,10,20,30,50,75,100,125,150$, and $200 \sigma$, with an $\mathrm{rms}=30 \mu \mathrm{Jy}$.

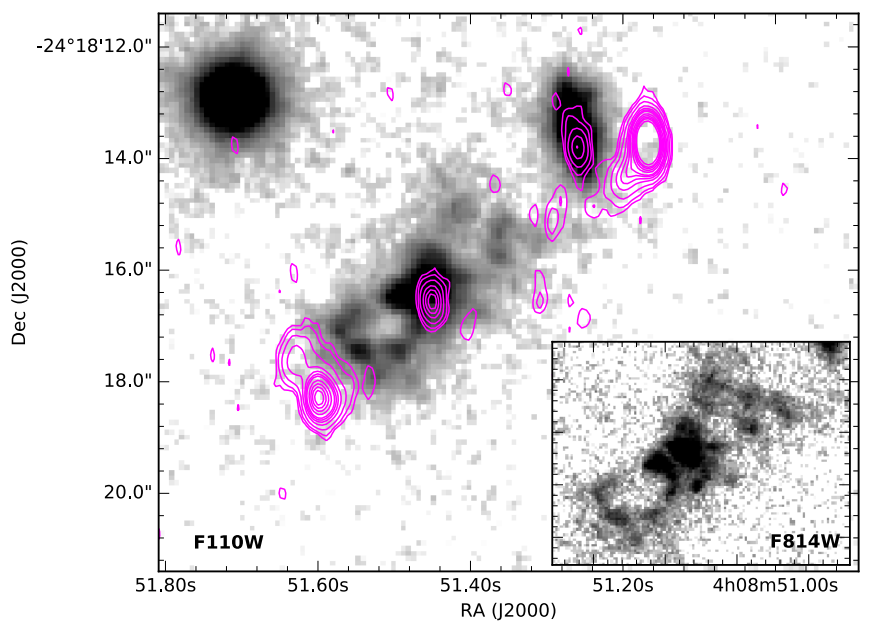

Fig. 13. Composite view of MRC 0406-244 $(z=2.43)$. The main frame is at $1.1 \mu \mathrm{m}$ HST image while the inset corresponds to the $I$ band (Hatch et al. 2013). The magenta contours are the $8.4 \mathrm{GHz}$ data overlaid at $3,5,10,20,30,50,75,100,125,150$, and $200 \sigma$, with an $\mathrm{rms}=30 \mu \mathrm{Jy}$. The source North-West of the radio galaxy is a foreground source (Rush et al. 1997).

The nuclear starburst (star formation within the central $1 \mathrm{kpc}$ ) might be expected and related to the AGN activity as they both require gas. To trigger a bolometrically luminous phase, an SMBH needs to accrete at rates of the order of $10 M_{\odot} \mathrm{yr}^{-1}$ (e.g. Drouart et al. 2014). This implies that cold gas needs to be present in the central parts of radio galaxies. Because most of the gas is not expected to directly fall onto the black hole (the gas

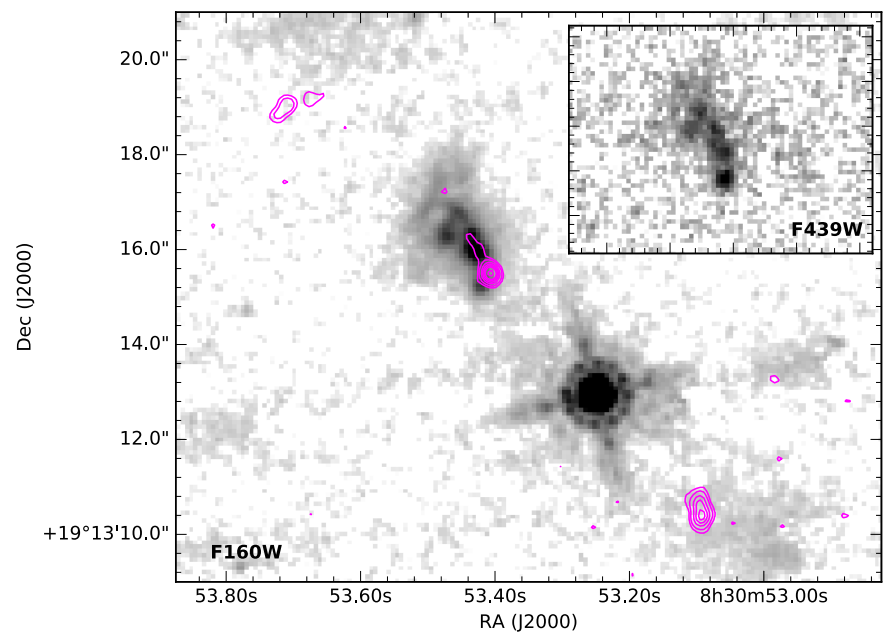

Fig. 14. Composite view of USS $0828+193(z=2.572)$. The main frame is at $1.6 \mathrm{HST} \mu \mathrm{m}$ image while the inset corresponds to the $u$ band. The magenta contours are the $8.4 \mathrm{GHz}$ data overlaid at $3,5,10,20,30,50$, $75,100,125,150$, and $200 \sigma$, with an rms $=40 \mu \mathrm{Jy}$. The source located South-West of the radio galaxy.

has to lose $>99 \%$ of its angular momentum, Jogee 2006), large reservoirs are expected to accumulate close to the centre of the galaxy. Collapse of the gas clouds is therefore likely to occur, producing central star formation. This element seems to agree with the correlation found between AGN activity and radial star formation dependency (e.g., Asmus et al. 2011; Videla et al. 2013; Esquej et al. 2014). However this relation between SB and AGN luminosities is still highly debated and might not hold at higher redshift (e.g. Dicken et al. 2012; Drouart et al. 2014). In this sample, our limited range of luminosity both for the SB and the AGN prevents us from finding any correlation, which suggests a weak correlation if it exists at all.

Several arguments in favour of a central nuclear starburst for these three source come from the SED fitting: the $K=10$ (higher column density), the short timescale ( $\delta$-like function as used here), and the massive starburst required to reproduce the large submm emission. In such constrained volume, a fast depletion of the gas is expected and required to create high star formation rates. Our discussion on the origin of the nuclear starburst overlaps with Sect. 5.3.3 because an AGN could trigger a nuclear starburst through intense activity (e.g. Silk 2013). In this case, the difference between AGN-driven star formation and secular star formation (not triggered by the AGN) is difficult to assess because higher resolution observation are crucial to distinguish between these two cases.

\subsubsection{AGN-driven star formation}

We include in this category star formation triggered from AGN activity, would it be mechanical and/or radiative (radio jets, UV, optical, and infrared radiation). The so-called alignment effect in radio galaxies was observed more than two decades ago, and jet-induced star formation was proposed as a possible explanation (e.g., McCarthy et al. 1987; Miley et al. 1992; Chambers et al. 1996a; van Breugel et al. 1998; Pentericci et al. 1999; Zirm et al. 2003). We need to be careful, however, in associating elongated UV emission with star formation because other mechanisms can produce similar clumpy structures in high-resolution observations (e.g., 


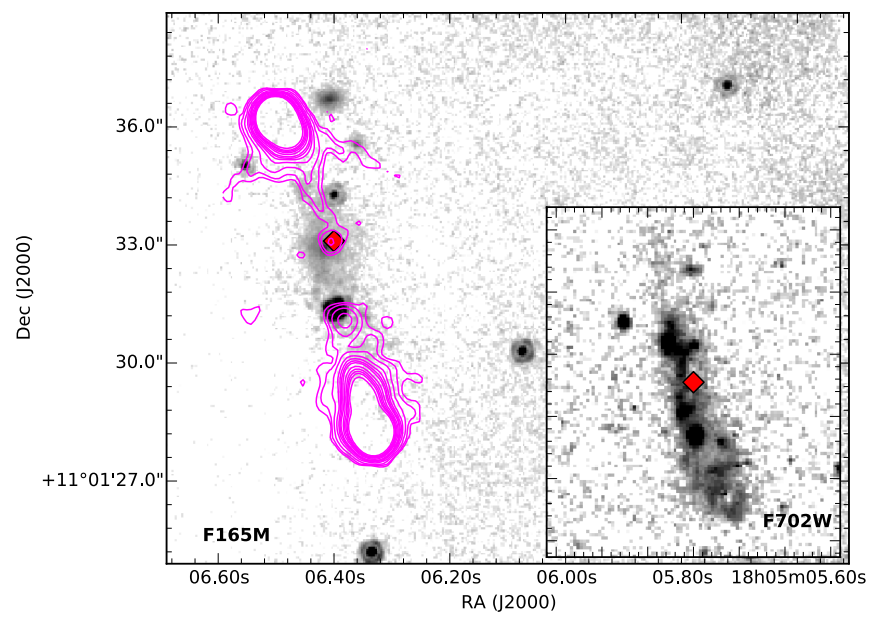

Fig. 15. Composite view of $3 C 368(z=1.132)$. The main frame is a $1.6 \mu \mathrm{m}$ HST image while the inset corresponds to the $R$ band. Two stars are located at $\sim 2^{\prime \prime}$. The magenta contours show the $4.8 \mathrm{GHz}$ data overlaid at $3,5,10,20,30,50,75,100,125,150$, and $200 \sigma$, with an $\mathrm{rms}=20 \mu \mathrm{Jy}$.

Dickson et al. 1995; Chambers et al. 1996a; Cimatti et al. 1998; van Breugel et al. 1998; Pentericci et al. 1999). The other likely candidates for the origin of such emission are either scattered emission from the AGN (Cimatti et al. 1998) and direct nebular emission (excited either from radiative or mechanical shocks; Humphrey et al. 2006; Villar-Martín et al. 2007). One simple test to distinguish between AGN-scattered emission and nebular emission are polarimetric measurements. Strongly polarised sources will be AGN dominated, whereas weakly polarised sources will be dominated by nebular and/or young stars (e.g., Vernet et al. 2001, Sect. 4.4).

In our sample, we focused on the three sources with the richest data sets, 3C 368 (Fig. 15), 4C 41.17 (Fig. 6) and MRC 0406244 (Fig. 13), where the AGN may have a limited effect on star formation. 3C 368 presents striking elongated features aligned with the radio emission (Best et al. 1998). The source is also moderately polarised $(7.6 \%)$, with a wide range of contributions from $20 \%$ to $80 \%$ from the AGN-scattered light. This makes it particularly difficult to assess the origin of the emission, but given the low mass of the starburst compared to the total mass of the system, star formation along the jet axis may be possible. For 4C 41.17, observations provide strong evidence that a massive star-forming region is aligned with the radio jet axis (Miley et al. 1992; Dey et al. 1997; Bicknell et al. 2000; Steinbring 2014), and polarisation for this source is weak $<2.4 \%$. The source 4C 41.17 appears to be an exceptional case even among radio galaxies because a large molecular cloud is aligned with the radio jet axis (De Breuck et al. 2005). However, recent mm observations suggest that this fortuitous alignment, although rare, may not be unique (Emonts et al. 2014). Finally, in MRC 0406-244 (weakly polarised $P=2.5 \%$ ), Hatch et al. (2013) found structures along the jet axis, implying an SFR of $\sim 75 M_{\odot} \mathrm{yr}^{-1}$ assuming it is due to star formation and not scattered AGN emission.

It seems that AGN-driven star formation, even if not dominant in our sample, does contribute to some extent. This is in line with a a comparison of radio-loud and radio-quiet AGN that found higher average SFRs for radio-loud AGN (Zinn et al. 2013) which does suggest an effect of the jet on star formation (though also see Karouzos et al. 2014, for an opposite conclusion). When we use the information available from the SED fitting and observational constraints, we are able to predict some of the properties of an SB triggered by a jet. As seen in the last line of Table 5, when half of the mass of the SB component is contained in a region of $1 \mathrm{kpc}$, the dynamical time is of the order of $10^{6} \mathrm{yr}$ and the sSFR is higher by a factor of $\sim 20$ compared to the rest of the sample. This short and very efficient star formation makes sense in such an extremely contained volume, where as the gas will be expelled extremely quickly and efficiently, as soon as the first SN explode, which will terminate the episode of star formation.

\section{Conclusion}

We presented 11 objects from the HeRGÉ sample, selected to obtain a homogeneous coverage over the UV to submm range. We performed SED fitting with the PÉGASE.3 galaxy evolution and the Fritz et al. (2006) AGN torus models, both of which use radiative transfer code to predict their respective SEDs. We showed that the SEDs of high-redshift radio galaxies require three spectral components: two stellar components, one evolved and one young, plus an AGN torus.

All evolved components are very massive, $>10^{11} M_{\odot}$, and are consistent with formation at high-redshift. The young component represents up to $61 \%$ of the total mass of the system and is much younger than the evolved component, indicating on-going or recently completed star formation. The physical properties of these starbursts broadly agree with SMGs at similar redshift and present a large variation from source to source. The radio galaxies are generally in a luminous phase with a preferentially extended, opaque torus, consistent with a type $2 \mathrm{AGN}$ orientation. The bolometric luminosities are similar to quasars at similar redshift but the AGN activity observed at radio and optical wavelengths does not appear to be strongly correlated with the star formation within our sample. We also considered reflected AGN contamination in our SED fitting based on polarisation measurements for seven sources in this sample and found that for broadband photometry, fitting the differences induced by prior subtraction of the reflected AGN falls within the confidence intervals of the fitting and does not strongly affect the results.

Finally, our sample contains evidence of ex situ star formation, with $65 \%$ of the sources presenting evidence of merging activity. In addition, $30-55 \%$ of the sources also present in situ star formation, indicating large amounts of gas accreted directly by the galaxy. Some weak evidence for jet-induced star formation (although rather limited in terms of mass and relative bolometric contribution) appears in $35 \%$ of our sample. An additional analysis, including high-resolution submm observations and optical/NIR integral field maps, will allow characterisation of emission and absorption lines and will most likely bring new insights.

Acknowledgements. G.D. would like to warmly thank Alessandro Romeo, Kirsten Knudsen, and Clive Tadhunter for the useful discussions that contributed to improve this paper. The authors also thank the referee for detailed suggestions and a thorough report that helped to clarify this paper. G.D. also thanks Nina Hatch for providing HST fluxes for the Spiderweb galaxy and A. Galametz for providing images for part of the sample. G.D. thanks Philip Best for providing the 3C 368 and 3C 470 data. N.S. is the recipient of an ARC Future Fellowship. The work of DS was carried out at Jet Propulsion Laboratory, California Institute of Technology, under a contract with NASA. Based on observations made with the NASA/ESA Hubble Space Telescope, and obtained from the Hubble Legacy Archive, which is a collaboration between the Space Telescope Science Institute (STScI/NASA), the Space Telescope European Coordinating Facility (ST-ECF/ESA) and the Canadian Astronomy Data Centre (CADC/NRC/CSA). GD acknowledges the support from the ESO scientific visitor programme. 


\section{References}

Akiyama, M., Minowa, Y., Kobayashi, N., et al. 2008, ApJS, 175, 1 Alexander, D. M., Smail, I., Bauer, F. E., et al. 2005, Nature, 434, 738 Antonucci, R. 1993, ARA\&A, 31, 473

Antonucci, R. 2012, Astron. Astrophys. Trans., 27, 557

Archibald, E. N., Dunlop, J. S., Hughes, D. H., et al. 2001, MNRAS, 323, 417

Asensio Ramos, A., \& Ramos Almeida, C. 2009, ApJ, 696, 2075

Asmus, D., Gandhi, P., Smette, A., Hönig, S. F., \& Duschl, W. J. 2011, A\&A, 536, A36

Banerji, M., Fabian, A. C., \& McMahon, R. G. 2014, MNRAS, 439, L51

Barthel, P. D. 1989, ApJ, 336, 606

Bastian, N., Covey, K. R., \& Meyer, M. R. 2010, ARA\&A, 48, 339

Best, P. N., Longair, M. S., \& Roettgering, J. H. A. 1997, MNRAS, 292, 758

Best, P. N., Carilli, C. L., Garrington, S. T., Longair, M. S., \& Rottgering, H. J. A 1998, MNRAS, 299, 357

Bicknell, G. V., Sutherland, R. S., van Breugel, W. J. M., et al. 2000, ApJ, 540, 678

Bigiel, F., Leroy, A., Walter, F., et al. 2008, AJ, 136, 2846

Blain, A. W., Smail, I., Ivison, R. J., \& Kneib, J.-P. 1999, MNRAS, 302, 632

Bornancini, C. G., De Breuck, C., de Vries, W., et al. 2007, MNRAS, 378, 551

Borys, C., Chapman, S., Halpern, M., \& Scott, D. 2003, MNRAS, 344, 385

Bruzual, G., \& Charlot, S. 2003, MNRAS, 344, 1000

Buchard, D. 2008, Master's thesis

Burgarella, D., Buat, V., \& Iglesias-Páramo, J. 2005, MNRAS, 360, 1413

Chambers, K. C., Miley, G. K., \& van Breugel, W. 1987, Nature, 329, 604

Chambers, K. C., Miley, G. K., \& van Breugel, W. J. M. 1990, ApJ, 363, 21

Chambers, K. C., Miley, G. K., van Breugel, W. J. M., et al. 1996a, ApJS, 106, 247

Chambers, K. C., Miley, G. K., van Breugel, W. J. M., \& Huang, J.-S. 1996b, ApJS, 106, 215

Chary, R., \& Elbaz, D. 2001, ApJ, 556, 562

Chen, C.-C., Smail, I., Swinbank, A. M., et al. 2015, ApJ, 799, 194

Chini, R., \& Kruegel, E. 1994, A\&A, 288, L33

Ciesla, L., Charmandaris, V., Georgakakis, A., et al. 2015, A\&A, 576, A10

Cimatti, A., di Serego Alighieri, S., Vernet, J., Cohen, M., \& Fosbury, R. A. E. 1998, ApJ, 499, L21

Conroy, C. 2013, ARA\&A, 51, 393

Coppin, K., Chapin, E. L., Mortier, A. M. J., et al. 2006, MNRAS, 372, 1621

Cristiani, S., \& Vio, R. 1990, A\&A, 227, 385

da Cunha, E., Charlot, S., \& Elbaz, D. 2008, MNRAS, 388, 1595

De Breuck, C., van Breugel, W., Stanford, S. A., et al. 2002, AJ, 123, 637

De Breuck, C., Downes, D., Neri, R., et al. 2005, A\&A, 430, L1

De Breuck, C., Seymour, N., Stern, D., et al. 2010, ApJ, 725, 36

Dey, A., Spinrad, H., \& Dickinson, M. 1995, ApJ, 440, 515

Dey, A., van Breugel, W., Vacca, W. D., \& Antonucci, R. 1997, ApJ, 490, 698

di Serego Alighieri, S., Fosbury, R. A. E., Tadhunter, C. N., \& Quinn, P. J. 1989, Nature, 341, 307

di Serego Alighieri, S., Cimatti, A., Fosbury, R. A. E., \& Perez-Fournon, I. 1996, MNRAS, 279, L57

Djorgovski, S., Spinrad, H., Pedelty, J., Rudnick, L., \& Stockton, A. 1987, AJ, 93, 1307

Dickson, R., Tadhunter, C., Shaw, M., Clark, N., \& Morganti, R. 1995, MNRAS, 273, L29

Dicken, D., Tadhunter, C., Axon, D., et al. 2009, ApJ, 694, 268

Dicken, D., Tadhunter, C., Axon, D., et al. 2012, ApJ, 745, 172

Doherty, M., Tanaka, M., De Breuck, C., et al. 2010, A\&A, 509, A83

Draine, B. T. 2003, ARA\&A, 41, 241

Drouart, G., De Breuck, C., Vernet, J., et al. 2012, A\&A, 548, A45

Drouart, G., De Breuck, C., Vernet, J., et al. 2014, A\&A, 566, A53

Dullemond, C. P., \& van Bemmel, I. M. 2005, A\&A, 436, 47

Dunlop, J. S., Hughes, D. H., Rawlings, S., Eales, S. A., \& Ward, M. J. 1994, Nature, 370, 347

Elbaz, D., Dickinson, M., Hwang, H. S., et al. 2011, A\&A, 533, A119

Emonts, B. H. C., Norris, R. P., Feain, I., et al. 2014, MNRAS, 438, 2898

Emonts, B. H. C., De Breuck, C., Lehnert, M. D., et al. 2015, A\&A, 584, A99

Esquej, P., Alonso-Herrero, A., González-Martín, O., et al. 2014, ApJ, 780, 86

Fan, L., Lapi, A., Bressan, A., et al. 2010, ApJ, 718, 1460

Feltre, A., Hatziminaoglou, E., Fritz, J., \& Franceschini, A. 2012, MNRAS, 426 120

Feltre, A., Hatziminaoglou, E., Hernán-Caballero, A., et al. 2013, MNRAS, 434, 2426

Ferrarese, L., \& Merritt, D. 2000, ApJ, 539, L9

Fioc, M., \& Rocca-Volmerange, B. 1997, A\&A, 326, 950

Fritz, J., Franceschini, A., \& Hatziminaoglou, E. 2006, MNRAS, 366, 767

Galametz, A., Stern, D., De Breuck, C., et al. 2012, ApJ, 749, 169

Gebhardt, K., Bender, R., Bower, G., et al. 2000, ApJ, 539, L13

Greve, T. R., Stern, D., Ivison, R. J., et al. 2007, MNRAS, 382, 48
Groves, B., Dopita, M. A., Sutherland, R. S., et al. 2008, ApJS, 176, 438 Gullberg, B., De Breuck, C., Lehnert, M. D., et al. 2016, A\&A, 586, A124 Haas, M., Willner, S. P., Heymann, F., et al. 2008, ApJ, 688, 122 Häring, N., \& Rix, H.-W. 2004, ApJ, 604, L89

Hatch, N. A., Overzier, R. A., Kurk, J. D., et al. 2009, MNRAS, 395, 114 Hatch, N. A., Kurk, J. D., Pentericci, L., et al. 2011, MNRAS, 415, 2993 Hatch, N. A., Röttgering, H. J. A., Miley, G. K., et al. 2013, MNRAS, 436, 2244 Hewitt, A., \& Burbidge, G. 1991, ApJS, 75, 297

Holland, W. S., Robson, E. I., Gear, W. K., et al. 1999, MNRAS, 303, 659 Hönig, S. F., Beckert, T., Ohnaka, K., \& Weigelt, G. 2006, A\&A, 452, 459 Humphrey, A., Villar-Martín, M., Fosbury, R., Vernet, J., \& di Serego Alighieri, S. 2006, MNRAS, 369, 1103

Ilbert, O., McCracken, H. J., Le Fèvre, O., et al. 2013, A\&A, 556, A55 Inskip, K. J., Best, P. N., Longair, M. S., \& MacKay, D. J. C. 2002, MNRAS, 329,277

Inskip, K. J., Best, P. N., \& Longair, M. S. 2006, MNRAS, 367, 693

Ivison, R. J., Morrison, G. E., Biggs, A. D., et al. 2008, MNRAS, 390, 1117

Ivison, R. J., Smail, I., Amblard, A., et al. 2012, MNRAS, 425, 1320

Jogee, S. 2006, in Physics of Active Galactic Nuclei at all Scales ed. D. Alloin (Berlin Springer Verlag), Lect. Notes Phys., 693, 143

Karouzos, M., Im, M., Trichas, M., et al. 2014, ApJ, 784, 137

Kartaltepe, J. S., Sanders, D. B., Le Floc'h, E., et al. 2010, ApJ, 709, 572

Knopp, G. P., \& Chambers, K. C. 1997, ApJ, 487, 644

Kollmeier, J. A., Onken, C. A., Kochanek, C. S., et al. 2006, ApJ, 648, 128

Kroupa, P. 2001, MNRAS, 322, 231

Kuiper, E., Hatch, N. A., Röttgering, H. J. A., et al. 2010, MNRAS, 405, 969

Le Borgne, D., \& Rocca-Volmerange, B. 2002, A\&A, 386, 446

Lehnert, M. D., \& Heckman, T. M. 1996, ApJ, 472, 546

Leitherer, C., \& Heckman, T. M. 1995, ApJS, 96, 9

Leitherer, C., Schaerer, D., Goldader, J. D., et al. 1999, ApJS, 123, 3

Leipski, C., Haas, M., Willner, S. P., et al. 2010, ApJ, 717, 766

Leipski, C., Meisenheimer, K., Walter, F., et al. 2014, ApJ, 785, 154

Lilly, S. J., \& Longair, M. S. 1984, MNRAS, 211, 833

Magorrian, J., Tremaine, S., Richstone, D., et al. 1998, AJ, 115, 2285

Maraston, C. 1998, MNRAS, 300, 872

Maraston, C. 2005, MNRAS, 362, 799

Marchesini, D., van Dokkum, P. G., Förster Schreiber, N. M., et al. 2009, ApJ, 701,1765

Mayo, J. H., Vernet, J., De Breuck, C., et al. 2012, A\&A, 539, A33

McCarthy, P. J. 1988, Ph.D. Thesis, California Univ., Berkeley

McCarthy, P. J. 1993, ARA\&A, 31, 639

McCarthy, P. J., van Breugel, W., Spinrad, H., \& Djorgovski, S. 1987, ApJ, 321, L29

McCarthy, P. J., Spinrad, H., Dickinson, M., et al. 1990, ApJ, 365, 487

McCarthy, P. J., van Breugel, W., \& Kapahi, V. K. 1991, ApJ, 371, 478

McCarthy, P. J., Baum, S. A., \& Spinrad, H. 1996, ApJS, 106, 281

Meisenheimer, K., \& Hippelein, H. 1992, A\&A, 264, 455

Meisenheimer, K., Haas, M., Müller, S. A. H., et al. 2001, A\&A, 372, 719

Miley, G., \& De Breuck, C. 2008, A\&ARv, 15, 67

Miley, G. K., Chambers, K. C., van Breugel, W. J. M., \& Macchetto, F. 1992, ApJ, 401, L69

Miley, G. K., Overzier, R. A., Zirm, A. W., et al. 2006, ApJ, 650, L29

Mullaney, J. R., Alexander, D. M., Goulding, A. D., \& Hickox, R. C. 2011, MNRAS, 414, 1082

Nenkova, M., Sirocky, M. M., Ivezić, Ž., \& Elitzur, M. 2008, ApJ, 685, 147

Noll, S., Burgarella, D., Giovannoli, E., et al. 2009, A\&A, 507, 1793

Pentericci, L., Roettgering, H. J. A., Miley, G. K., et al. 1998, ApJ, 504, 139

Pentericci, L., Röttgering, H. J. A., Miley, G. K., et al. 1999, A\&A, 341, 329

Pentericci, L., McCarthy, P. J., Röttgering, H. J. A., et al. 2001, ApJS, 135, 63

Pier, E. A., \& Krolik, J. H. 1992, ApJ, 401, 99

Pieri, M. M., Mortonson, M. J., Frank, S., et al. 2014, MNRAS, 441, 1718

Pilbratt, G. L., Riedinger, J. R., Passvogel, T., et al. 2010, A\&A, 518, L1

Popesso, P., Magnelli, B., Buttiglione, S., et al. 2012, ArXiv e-prints [arXiv: 1211.4257]

Press, W. H., Teukolsky, S. A., Vetterling, W. T., \& Flannery, B. P. 1992, Numerical recipes in FORTRAN, The art of scientific computing

Rawlings, J. I., Seymour, N., Page, M. J., et al. 2013, MNRAS, 429, 744

Reuland, M., Röttgering, H., van Breugel, W., \& De Breuck, C. 2004, MNRAS, 353,377

Rix, H.-W., de Zeeuw, P. T., Cretton, N., van der Marel, R. P., \& Carollo, C. M. 1997, ApJ, 488, 702

Rocca-Volmerange, B., Le Borgne, D., De Breuck, C., Fioc, M., \& Moy, E. 2004, A\&A, 415, 931

Rocca-Volmerange, B., Drouart, G., De Breuck, C., et al. 2013, MNRAS, 429, 2780

Rodighiero, G., Daddi, E., Baronchelli, I., et al. 2011, ApJ, 739, L40

Roettgering, H. J. A., van Ojik, R., Miley, G. K., et al. 1997, A\&A, 326, 505 
Rosario, D. J., Santini, P., Lutz, D., et al. 2012, A\&A, 545, A45

Rottgering, H. J. A., Hunstead, R. W., Miley, G. K., van Ojik, R., \& Wieringa, M. H. 1995, MNRAS, 277, 389

Rush, B., McCarthy, P. J., Athreya, R. M., \& Persson, S. E. 1997, ApJ, 484, 163 Salpeter, E. E. 1955, ApJ, 121, 161

Sanders, D. B., \& Mirabel, I. F. 1996, ARA\&A, 34, 749

Seymour, N., Stern, D., De Breuck, C., et al. 2007, ApJS, 171, 353

Seymour, N., Altieri, B., De Breuck, C., et al. 2012, ApJ, 755, 146 Silk, J. 2013, ApJ, 772, 112

Siringo, G., Kreysa, E., Kovács, A., et al. 2009, A\&A, 497, 945

Spergel, D. N., Verde, L., Peiris, H. V., et al. 2003, ApJS, 148, 175

Stacey, G. J., Hailey-Dunsheath, S., Ferkinhoff, C., et al. 2010, ApJ, 724, 957

Stalevski, M., Fritz, J., Baes, M., Nakos, T., \& Popović, L. Č. 2012, MNRAS, 420,2756

Steinbring, E. 2014, AJ, 148, 10

Steinbring, E., Crampton, D., \& Hutchings, J. B. 2002, ApJ, 569, 611

Stevens, J. A., Ivison, R. J., Dunlop, J. S., et al. 2003, Nature, 425, 264

Stockton, A., Ridgway, S. E., \& Kellogg, M. 1996, AJ, 112, 902

Symeonidis, M., Vaccari, M., Berta, S., et al. 2013, MNRAS, 431, 2317
Tadhunter, C., Dicken, D., Holt, J., et al. 2007, ApJ, 661, L13

Targett, T. A., Dunlop, J. S., McLure, R. J., et al. 2011, MNRAS, 412, 295

Trichas, M., Green, P. J., Silverman, J. D., et al. 2012, ApJS, 200, 17

Tsalmantza, P., Kontizas, M., Rocca-Volmerange, B., et al. 2009, A\&A, 504, 1071

Tsalmantza, P., Karampelas, A., Kontizas, M., et al. 2012, A\&A, 537, A42

van Breugel, W. J. M., Stanford, S. A., Spinrad, H., Stern, D., \& Graham, J. R. 1998, ApJ, 502, 614

van Ojik, R. 1995, in Ph.D. Thesis, Sterrewacht Leiden, 29

Vernet, J., Fosbury, R. A. E., Villar-Martín, M., et al. 2001, A\&A, 366, 7

Videla, L., Lira, P., Andrews, H., et al. 2013, ApJS, 204, 23

Villar-Martín, M., Sánchez, S. F., Humphrey, A., et al. 2007, MNRAS, 378, 416

Wang, R., Wagg, J., Carilli, C. L., et al. 2011, AJ, 142, 101

Weiß, A., Kovács, A., Coppin, K., et al. 2009, ApJ, 707, 1201

Werner, M. W., Roellig, T. L., Low, F. J., et al. 2004, ApJS, 154, 1

Wylezalek, D., Galametz, A., Stern, D., et al. 2013a, ApJ, 769, 79

Wylezalek, D., Vernet, J., De Breuck, C., et al. 2013b, MNRAS, 428, 3206

Zinn, P.-C., Middelberg, E., Norris, R. P., \& Dettmar, R.-J. 2013, ApJ, 774, 66

Zirm, A. W., Dickinson, M., \& Dey, A. 2003, ApJ, 585, 90 


\section{Appendix A: Systematics}

A great part of the systematics in our analysis is inherent to the code and the various approximations and assumptions made to build the stellar evolution tracks, metal enrichment, SN rates, etc. We discuss here only the internal systematics in PÉGASE. For a comparison between models, we refer to Conroy 2013. In the case of PÉGASE, the choice of IMF and star formation law certainly produce systematics that can affect our results.

The IMF has been subject to considerable discussion, particularly concerning the potential universality of the IMF (see Bastian et al. 2010, for a recent review). The difference in mass between IMF between a Salpeter (1955) IMF and a Kroupa (2001) IMF is contained in a factor of $\sim 2$, as noted in RV13 and other studies (e.g. Marchesini et al. 2009).

As for the star formation law, the PÉGASE code sets the star formation history and star formation efficiency based on the Hubble type galaxies at $z=0$. To test how sensitive our results are to this choice, we used four scenarios for the host galaxy (evolved component): E, E2, S0, and Sa templates (Fioc et al., in prep.). While the results show variations (up to 4 in mass and age, $\sim 2$ in most cases), it is impossible to select a preferred scenario based on the $\chi^{2}$ values. The star formation history of the evolved component is therefore our largest systematic. That said, even if mass and age present variations, the fact that two stellar components are needed to reproduce the SED (see Sect. 4) remains unchanged and does not affect the primary conclusions of this paper.

The main systematics for the AGN component are the continuous approach and radial and azimuthal dust density profiles. For the clumpy versus smooth tori, Dullemond \& van Bemmel (2005) and Feltre et al. (2012) showed that they can be equivalent for a given set of parameters. Fritz et al. (2006) showed that the dust density profiles have a limited effect on the continuum properties in our considered range (at most 10\%, see their Figs. 7 and 8).

\section{Appendix B: Domain, interval, and weight of the parameters}

Each parameter was defined onto a grid, presented in Table 2. Only the stellar masses and the AGN luminosity were calculated directly from the normalisation of the components during the fitting and were an output of our SED fitting. However, each parameter has a different effect on the final SED. They were also constrained mostly in the wavelength range they dominate. For instance, a type 2 AGN consists of an accreting SMBH obscured by a dusty torus, with mostly high dust temperature $(>200 \mathrm{~K})$, and it mostly dominates in the mid-IR domain. Therefore good mid-IR data will allow constraining better the parameters of the AGN model better. We discuss this weight of the parameters and their final effect on the broad-band SED.

\section{B.1. AGN component}

As mentioned previously, we focused on typical configurations of the AGN torus. The size is important because the farther out the central part, the cooler the dust, causing the SED to peak at longer wavelength. Opacity also plays a role in the overall AGN SED in the IR. In particular, the opacity affects both the peak of the dust emission and its width (see Figs. 7 and 8 of Fritz et al. 2006). These parameters mostly affect the transition regime from dust heated by the AGN to dust heated by the
$\mathrm{SB}$, in the $40-60 \mu \mathrm{m}$ range. We note that some of our results show a discrepancy in this wavelength range, suggesting that the torus size is not sufficiently explored in our fitting procedure (see Sect. 5.1). The AGN inclination will have the strongest effect at shorter wavelength, affecting the peak of the dust emission (typically $\sim 4-10 \mu \mathrm{m}$ restframe), where the fraction of the view of the innermost part of the AGN (i.e. close the sublimation radius) is visible to the observer increases. This wavelength range is particularly critical because it concerns the transition between the emission of intermediate-to-low-mass stars, evolved stars (e.g. AGB) and the host dust of the AGN torus. We therefore selected three torus sizes, with $Y=10, Y=60$ and $Y=150$, which represent different extensions of the outer part of the torus, to test the effect of the torus size on the AGN-SB transition $(\sim 40-60 \mu \mathrm{m}$ restframe interval).

\section{B.2. PÉGASE components}

For the SB component, mass and age are among the most important parameters (see Fig. 2 of RV13) as both parameters strongly affect the luminosity and shape of the SED. A young stellar population produces UV and strong dust heating, the mass of the system and a rapid metal enrichment when the most massive stars eventually explode. The column density is also of great importance, as a higher column density implies more absorption by dust. The direct effect of this is will be a shift of the SB dust peak to higher temperature (i.e. shorter wavelength) and decreased UV and optical emission. The two highest redshift sources of our sample, presented in RV13, show a clear preference for a higher column density factor to reproduce the SED $(K=10)$, confirmed in this analysis. The initial metallicity is the parameter with the most limited effect on the SED in this analysis. However, the difference between a null metallicity and a highly enriched initial gas implies a strong change on the stellar evolutionary tracks, and therefore an effect on the other parameters and on the final SED. Spectroscopic data are required to fully assess this parameter, while broad-band photometry provides very limited constraints on this parameter. However, we kept this parameter in our fitting to determine the final effect on our best fit and whether a complete fitting from UV to submm can provide useful information on the initial metallicity.

For the evolved component, we recall that PÉGASE predicts the integrated SED for a given scenario, and the Hubble type refers, for convenience, to the combination of parameters necessary to reproduce the observed colours or SED of the classical Hubble type galaxies at $z=0$. The model assumes a closedbox evolution (with a galaxy plus reservoir), where progressive enrichment of the ISM translates into more dust. The main parameters are $z_{\text {form }}$, the astration, the star formation rate, the infall timescale and the galactic winds. In addition, each galaxy type corresponds to a different set of these parameters. By considering several types, we explore different star formation histories that will reproduce observed galaxies in the local Universe (Tsalmantza et al. 2009, 2012). The initial metallicity is null as galaxies are assumed to form from primordial, metal-free gas as formed at high redshift $z_{\text {form }}=10$. A slight change of $z_{\text {form }}$ will not have a dramatic effect because the fraction of age at high redshift is not significant compared to the age at $z=0$. The star formation rate is proportional to the gas available in the galaxy, the gas supply being driven by the infall timescale. A longer infall timescale, such as the spiral scenario, allows more moderate star formation over longer periods, reproducing the star-forming disc, hence the galaxy appears bluer at optical 
wavelengths. The astration rate is the efficiency of converting gas into stars, increasing from spiral to elliptical. The galactic winds expel all the ISM from the galaxy, and thus prevent any further star formation. This parameter is only used in elliptical scenarios to reproduce the red colours of elliptical galaxies in the local Universe. Complete details of each parameter are described in Fioc \& Rocca-Volmerange (1997) for PÉGASE.2 and Fioc et al. (in prep.) for PÉGASE.3.

\section{Appendix C: Notes on individual sources}

We briefly summarise for each object the morphological peculiarities and interesting aspects from a multi-wavelength point of view. Each of the objects is extensively described in their respective papers, to which we refer for more details (see Tables D.1-D.11). We present the the NIR and optical data, along with radio contours when data are available in Sect. 5 from Fig. 6 to Fig. 15.

3C 368, in Fig. 15 - This galaxy is located a $z=1.13$ (Djorgovski et al. 1987), and has been extensively studied from X-ray to radio energies (e.g., Best et al. 1997; Zirm et al. 2003; Reuland et al. 2004; Leipski et al. 2010; Stacey et al. 2010). Two stars visible in high-resolution imaging (Best et al. 1997) could affect the final photometry presented in Table D.1. Best et al. (1997) identified two components assumed to be from the same galaxy. Between the optical and NIR images, the emission peak shifts to the north, implying that the northern component is likely heavily obscured (Stockton et al. 1996). The contour of the radio emission is aligned between the optical morphology and the jet axis.

3C 470, in Fig. 9 - This galaxy is located at $z=1.65$ (McCarthy 1988), and shows a potential companion in the highresolution optical image (Best et al. 1997) that also affects the photometry. For simplicity, we use photometry including the two components since the components blend at longer wavelength (i.e., the Herschel data). We note that the two components are not aligned with the radio jet axis, in contrast to most HzRGs (Chambers et al. 1996a; van Breugel et al. 1998).

MRC 0324-228, in Fig. $12-$ This galaxy is at $z=1.89$ (McCarthy et al. 1991) and has a companion in high-resolution NIR images (Pentericci et al. 2001). However, nothing indicates that this companion is part of the system.

PKS 1138-262, in Fig. 10 - Also named the "Spiderweb galaxy", this galaxy is at $z=2.16$ (van Ojik 1995). Its unusual morphology consists of a central massive galaxy surrounded by fainter companions (Pentericci et al. 1998; Miley et al. 2006; Kuiper et al. 2010; Hatch et al. 2011; Seymour et al. 2012). The galaxy has an extension that is aligned with the radio jet axis.

MRC 0406-244, in Fig. $13-$ This galaxy is at $z=2.43$ (McCarthy et al. 1991) and has an extensive data set (Rush et al. 1997). Some features, aligned with the radio jet axis in high-resolution optical images (Pentericci et al. 2001), suggest a complex system. New HST high-resolution data are also available (Hatch et al. 2013), enabling a more precise multiwavelength morphological characterisation. No companion is evident in the direct vicinity $\left(<3^{\prime \prime}\right)$ of the radio galaxy, and the ionisation cones are aligned with the radio jet axis.

MRC 2104-242, in Fig. 8 - This galaxy is at $z=2.49$ (McCarthy et al. 1991) and shows several components in the optical and NIR images (Pentericci et al. 2001). The images and spectroscopy exhibit a large Ly $\alpha$ halo extended along the radio lobes (Pentericci et al. 2001). A companion is present $\sim 2^{\prime \prime}$ north of the galaxy.

USS 0828+193, in Fig. $14-$ This source is at $z=2.57$ (Knopp \& Chambers 1997) and has a star $3^{\prime \prime}$ south of the galaxy, complicating the photometry. The radio galaxy itself seems to be split into multiple components in the high-resolution NIR images (Steinbring et al. 2002). The galaxy has polarimetric observations, that have been reported in Knopp \& Chambers (1997).

USS 0943-242, in Fig. 7 - This source is at $z=2.92$ (Rottgering et al. 1995) and has an elongation in the highresolution HST image that is broadly aligned with the radio jet axis. While the optical HST image does not show a close companion, the MIR IRAC image reveals a close potential companion 6 " south-west of the radio galaxy that appears at redder wavelengths. The two sources blend in the SPIRE images, $\lambda>250 \mu \mathrm{m}$.

4C 28.58 - This source is at $z=2.89$ (Chambers et al. $1996 \mathrm{~b})$ and presents a very disturbed morphology in HST images (Chambers et al. 1996a). Multiple components appear, the main flux coming from two components close to the radio core. Given astrometric uncertainties, we cannot determine which component is the primary radio galaxy. The elongation observed in the HST image is roughly aligned with the radio jet axis.

4C 41.17 and TN J2007-1316, in Figs. 6 and 11 - These sources, at $z=3.79$ and $z=3.84$, respectively, have been presented extensively in RV13. We briefly report some unusual properties. 4C 41.17 is weakly polarised (Dey et al. 1997), and shows a low contribution of the AGN in the IR (RV13). In addition, a star-forming companion is evident in the high-resolution optical images. TN J2007-1316 is also weakly polarised, but has a stronger AGN contribution in the MIR (RV13).

\section{Appendix D: Additional material: photometry and SED fit for the sample}

All Herschel flux are taken from Drouart et al. (2014). Some of these flux present a signal-to-noise ratio $<3$ and are explained by the method used to calculate photometry on our sources, from the $24 \mu \mathrm{m}$ prior and the calibration uncertainties. We refer to Sect. 2 in Drouart et al. (2014) for better details. 
G. Drouart et al.: Disentangling SF and AGN in luminous HzRGs

Table D.1. Data for 3C $368(z=1.132)$.

\begin{tabular}{lcccc}
\hline \hline Filter & $\begin{array}{c}\lambda_{0} \\
{[\mu \mathrm{m}]}\end{array}$ & $\begin{array}{c}\text { Line } \\
{[\%]}\end{array}$ & $\begin{array}{c}\text { Flux } \\
{[\mu \mathrm{Jy}]}\end{array}$ & Ref. \\
\hline B(CFHT) & 0.45 & - & $11.5 \pm 1.6$ & $\mathrm{~A}$ \\
R(CFHT) & 0.65 & - & $17.2 \pm 2.5$ & $\mathrm{~A}$ \\
F702W & 0.68 & 24 & $21.7 \pm 2.1$ & $\mathrm{~B}$ \\
F791W & 0.78 & 38 & $28.0 \pm 2.7$ & $\mathrm{~B}$ \\
J(UKIRT) & 1.27 & 3 & $36.9 \pm 4.0$ & $\mathrm{C}$ \\
H(UKIRT) & 1.67 & 2 & $71.6 \pm 9.1$ & $\mathrm{C}$ \\
K(UKIRT) & 2.2 & 4 & $85.0 \pm 8.2$ & $\mathrm{C}$ \\
IRAC1 & 3.6 & - & $126 \pm 13.0$ & $\mathrm{D}$ \\
IRAC2 & 4.5 & - & $112 \pm 11.0$ & $\mathrm{D}$ \\
IRAC3 & 5.8 & - & $112 \pm 11.0$ & $\mathrm{D}$ \\
IRAC4 & 8.0 & - & $210 \pm 21.0$ & $\mathrm{D}$ \\
IRS & 16.0 & - & $1620 \pm 180$ & $\mathrm{D}$ \\
MIPS1 & 24.0 & - & $3350 \pm 50.0$ & $\mathrm{D}$ \\
PACS 70 & 70 & - & $32300 \pm 3500$ & $\mathrm{E}$ \\
PACS 160 & 160 & - & $52900 \pm 9100$ & $\mathrm{E}$ \\
SPIRE 250 & 250 & - & $35600 \pm 6200$ & $\mathrm{E}$ \\
SPIRE 350 & 350 & - & $19600 \pm 6500$ & $\mathrm{E}$ \\
SPIRE 500 & 500 & - & $<19000$ & $\mathrm{E}$ \\
SCUBA 850 & 850 & - & $4080 \pm 1080$ & $\mathrm{~F}$ \\
IRAM 1300 & 1300 & - & $<600.0$ & $\mathrm{G}$ \\
\hline
\end{tabular}

Notes. $\lambda_{0}$ is the central wavelength, line contribution (in \%) in the broad-band, corrected in the final flux, flux in $\mathrm{mJy}$ (for a non-detection, the $3 \sigma$ upper limit is quoted).

References. (A) Djorgovski et al. (1987); (B) Best et al. (1997); (C) Inskip et al. (2006); (D) De Breuck et al. (2010); (E) Drouart et al. (2014); (F) Archibald et al. (2001); (G) Meisenheimer et al. (2001).

Table D.2. Data for 3C $470(z=1.65)$.

\begin{tabular}{lcccc}
\hline \hline Filter & $\begin{array}{c}\lambda_{0} \\
{[\mu \mathrm{m}]}\end{array}$ & $\begin{array}{c}\text { Line } \\
{[\%]}\end{array}$ & $\begin{array}{c}\text { Flux } \\
{[\mu \mathrm{Jy}]}\end{array}$ & Ref. \\
\hline F785LP & 0.87 & 15 & $4.71 \pm 1.5$ & $\mathrm{~A}$ \\
K(UKIRT) & 2.2 & - & $38.9 \pm 5.8$ & $\mathrm{~A}$ \\
K(UKIRT) & 2.2 & - & $35.8 \pm 4.2$ & $\mathrm{~B}$ \\
IRAC1 & 3.6 & - & $49.5 \pm 10.4$ & $\mathrm{C}$ \\
IRAC2 & 4.5 & - & $75.2 \pm 11.8$ & $\mathrm{C}$ \\
IRAC3 & 5.8 & - & $70.9 \pm 10.4$ & $\mathrm{C}$ \\
IRAC4 & 8.0 & - & $266 \pm 30.0$ & $\mathrm{C}$ \\
IRS & 16.0 & - & $1540 \pm 180$ & $\mathrm{C}$ \\
MIPS1 & 24.0 & - & $2750 \pm 40$ & $\mathrm{C}$ \\
PACS 70 & 70 & - & $21600 \pm 3700$ & $\mathrm{D}$ \\
PACS 160 & 160 & - & $22500 \pm 7200$ & $\mathrm{D}$ \\
SPIRE 250 & 250 & - & $39200 \pm 5700$ & $\mathrm{D}$ \\
SPIRE 350 & 350 & - & $33500 \pm 6000$ & $\mathrm{D}$ \\
SPIRE 500 & 500 & - & $24900 \pm 6300$ & $\mathrm{D}$ \\
SCUBA 850 & 850 & - & $<9300$ & $\mathrm{E}$ \\
\hline
\end{tabular}

Notes. Columns are the same as in Table D.1.

References. (A) Best et al. (1997); (B) Targett et al. (2011);

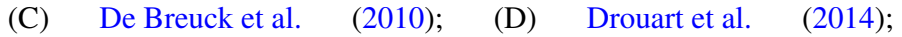
(E) Archibald et al. (2001).
Table D.3. Data for MRC 0324-228 $(z=1.894)$.

\begin{tabular}{lcccc}
\hline \hline Filter & $\begin{array}{c}\lambda_{0} \\
{[\mu \mathrm{m}]}\end{array}$ & $\begin{array}{c}\text { Line } \\
{[\%]}\end{array}$ & $\begin{array}{c}\text { Flux } \\
{[\mu \mathrm{Jy}]}\end{array}$ & Ref. \\
\hline R(Bessel) & 0.65 & - & $1.5 \pm 0.25$ & $\mathrm{~A}$ \\
F165M & 1.64 & - & $10.5 \pm 1.1$ & $\mathrm{~B}$ \\
IRAC1 & 3.6 & - & $39.4 \pm 4.2$ & $\mathrm{C}$ \\
IRAC2 & 4.5 & - & $39.7 \pm 4.3$ & $\mathrm{C}$ \\
IRAC3 & 5.8 & - & $61.1 \pm 8.6$ & $\mathrm{C}$ \\
IRAC4 & 8.0 & - & $89.9 \pm 9.9$ & $\mathrm{C}$ \\
IRS & 16.0 & - & $530.0 \pm 54.0$ & $\mathrm{C}$ \\
MIPS1 & 24.0 & - & $1880.0 \pm 35.0$ & $\mathrm{C}$ \\
PACS 70 & 70 & - & $<9100$ & $\mathrm{D}$ \\
PACS 160 & 160 & - & $27900 \pm 5400$ & $\mathrm{D}$ \\
SPIRE 250 & 250 & - & $61800 \pm 6700$ & $\mathrm{D}$ \\
SPIRE 350 & 350 & - & $35500 \pm 5900$ & $\mathrm{D}$ \\
SPIRE 500 & 500 & - & $17500 \pm 7400$ & $\mathrm{D}$ \\
LABOCA & 870 & - & $9000 \pm 2000$ & $\mathrm{D}$ \\
\hline
\end{tabular}

Notes. Columns are the same as in Table D.1.

References. (A) Buchard (2008); (B) Pentericci et al. (2001); (C) De Breuck et al. (2010); (D) Drouart et al. (2014).

Table D.4. Data for PKS 1138-262 $(z=2.156)$.

\begin{tabular}{lcccc}
\hline \hline Filter & $\begin{array}{c}\lambda_{0} \\
{[\mu \mathrm{m}]}\end{array}$ & $\begin{array}{c}\text { Line } \\
{[\%]}\end{array}$ & $\begin{array}{c}\text { Flux } \\
{[\mu \mathrm{Jy}]}\end{array}$ & Ref. \\
\hline$F 475 W$ & 0.48 & - & $7.66 \pm 0.8$ & N. Hatch (Priv.) \\
$F 814 W$ & 0.8 & - & $14.5 \pm 1.5$ & N. Hatch (Priv.) \\
$F 110 W$ & 1.1 & - & $29.4 \pm 3.0$ & N. Hatch (Priv.) \\
$F 160 W$ & 1.6 & - & $87.9 \pm 9.0$ & N. Hatch (Priv.) \\
Ks(ISAAC) & 2.16 & - & $207 \pm 21.0$ & N. Hatch (Priv.) \\
IRAC1 & 3.6 & - & $318 \pm 32.0$ & A \\
IRAC2 & 4.5 & - & $497 \pm 50.0$ & A \\
IRAC3 & 5.8 & - & $887 \pm 89.0$ & A \\
IRAC4 & 8.0 & - & $1500 \pm 150$ & A \\
IRS & 16.0 & - & $3020 \pm 100$ & A \\
MIPS1 & 24.0 & - & $3890 \pm 20$ & A \\
PACS 100 & 100 & - & $25200 \pm 2200$ & B \\
PACS 160 & 160 & - & $40200 \pm 10200$ & B \\
SPIRE 250 & 250 & - & $40400 \pm 5900$ & B \\
SPIRE 350 & 350 & - & $33000 \pm 6100$ & B \\
SPIRE 500 & 500 & - & $28900 \pm 6700$ & B \\
SCUBA 850 & 850 & - & $12800 \pm 3600$ & C \\
\hline
\end{tabular}

Notes. Columns are the same as in Table D.1. (Priv.) for "priv. comm.". References. (A) De Breuck et al. (2010); (B) Drouart et al. (2014); (C) Reuland et al. (2004). 
Table D.5. Data for MRC 0406-244 $(z=2.427)$.

\begin{tabular}{lcccc}
\hline \hline Filter & $\begin{array}{c}\lambda_{0} \\
{[\mu \mathrm{m}]}\end{array}$ & $\begin{array}{c}\text { Line } \\
{[\%]}\end{array}$ & $\begin{array}{c}\text { Flux } \\
{[\mu \mathrm{Jy}]}\end{array}$ & Ref. \\
\hline F555W & 0.52 & - & $2.11 \pm 0.31$ & $\mathrm{~A}$ \\
$r$ (SDSS) & 0.62 & - & $2.73 \pm 0.4$ & $\mathrm{~A}$ \\
$I$ (BESS) & 0.79 & - & $6.6 \pm 1.0$ & $\mathrm{~A}$ \\
$J$ (NIRC) & 1.25 & yes & $17.3 \pm 2.6$ & $\mathrm{~A}$ \\
$K$ (NIRC) & 2.2 & yes & $32.0 \pm 4.7$ & $\mathrm{~A}$ \\
IRAC1 & 3.6 & - & $40.4 \pm 4.3$ & $\mathrm{~B}$ \\
IRAC2 & 4.5 & - & $43.3 \pm 4.6$ & $\mathrm{~B}$ \\
IRAC3 & 5.8 & - & $<77.4$ & $\mathrm{~B}$ \\
IRAC4 & 8.0 & - & $63.5 \pm 14.5$ & $\mathrm{~B}$ \\
IRS & 16.0 & - & $637 \pm 86.0$ & $\mathrm{~B}$ \\
MIPS1 & 24.0 & - & $1540 \pm 40.0$ & $\mathrm{~B}$ \\
PACS 100 & 100 & - & $<12300$ & $\mathrm{C}$ \\
PACS 160 & 160 & - & $21500 \pm 7900$ & $\mathrm{C}$ \\
SPIRE 250 & 250 & - & $47600 \pm 5600$ & $\mathrm{C}$ \\
SPIRE 350 & 350 & - & $38700 \pm 5300$ & $\mathrm{C}$ \\
SPIRE 500 & 500 & - & $22800 \pm 5900$ & $\mathrm{C}$ \\
LABOCA & 870. & - & $<17800$ & $\mathrm{C}$ \\
\hline
\end{tabular}

Notes. Columns are the same as in Table D.1. No estimates available for the line contamination; so fluxes are not corrected. ${ }^{(*)}$ A galaxy NW might contaminate the photometry.

References. (A) Rush et al. (1997); (B) De Breuck et al. (2010); (C) Drouart et al. (2014).

Table D.6. Data for MRC 2104-242 $(z=2.49)$.

\begin{tabular}{lcccc}
\hline \hline Filter & $\begin{array}{c}\lambda_{0} \\
{[\mu \mathrm{m}]}\end{array}$ & $\begin{array}{c}\text { Line } \\
{[\%]}\end{array}$ & $\begin{array}{c}\text { Flux } \\
{[\mu \mathrm{Jy}]}\end{array}$ & Ref. \\
\hline F606W & 0.59 & - & $3.28 \pm 0.3$ & $\mathrm{~A}$ \\
$F 160 W$ & 1.6 & 26 & $29.5 \pm 3.8$ & $\mathrm{~B}$ \\
IRAC1 & 3.6 & - & $28.1 \pm 3.3$ & $\mathrm{C}$ \\
IRAC2 & 4.5 & - & $29.7 \pm 3.5$ & $\mathrm{C}$ \\
IRAC3 & 5.8 & - & $32.8 \pm 10.0$ & $\mathrm{C}$ \\
IRAC4 & 8.0 & - & $<54.4$ & $\mathrm{C}$ \\
IRS & 16.0 & - & $<325.5$ & $\mathrm{C}$ \\
MIPS1 & 24 & - & $709 \pm 48.0$ & $\mathrm{C}$ \\
PACS 100 & 100 & - & $14400 \pm 3500$ & $\mathrm{D}$ \\
PACS 160 & 160 & - & $22000 \pm 8400$ & $\mathrm{D}$ \\
SPIRE 250 & 250 & - & $14200 \pm 5100$ & $\mathrm{D}$ \\
SPIRE 350 & 350 & - & $21100 \pm 6600$ & $\mathrm{D}$ \\
SPIRE 500 & 500 & - & $<15800$ & $\mathrm{D}$ \\
\hline
\end{tabular}

Notes. Columns are the same as in Table D.1.

References. (A) Pentericci et al. (1999); (B) Pentericci et al. (2001); (C) De Breuck et al. (2010); (D) Drouart et al. (2014).
Table D.7. Data for USS $0828+193(z=2.57)$.

\begin{tabular}{lcccc}
\hline \hline Filter & $\begin{array}{c}\lambda_{0} \\
{[\mu \mathrm{m}]}\end{array}$ & $\begin{array}{c}\text { Line } \\
{[\%]}\end{array}$ & $\begin{array}{c}\text { Flux } \\
{[\mu \mathrm{Jy}]}\end{array}$ & Ref. \\
\hline$F 675 W$ & 0.59 & - & $1.51 \pm 0.15$ & $\mathrm{~A}$ \\
$J(\mathrm{KIR})$ & 1.25 & - & $15.2 \pm 1.5$ & $\mathrm{~A}$ \\
$H(\mathrm{KIR})$ & 1.63 & - & $36.1 \pm 3.5$ & $\mathrm{~A}$ \\
$K(\mathrm{KIR})$ & 2.2 & - & $51.2 \pm 8.9$ & $\mathrm{~A}$ \\
IRAC1 & 3.6 & - & $61.7 \pm 6.9$ & $\mathrm{~B}$ \\
IRAC2 & 4.5 & - & $133.0 \pm 13.0$ & $\mathrm{~B}$ \\
IRAC3 & 5.8 & - & $201.0 \pm 21.0$ & $\mathrm{~B}$ \\
IRAC4 & 8.0 & - & $687.0 \pm 74.0$ & $\mathrm{~B}$ \\
IRS & 16.0 & - & $1910 \pm 130$ & $\mathrm{~B}$ \\
MIPS1 & 24 & - & $2880 \pm 40.0$ & $\mathrm{~B}$ \\
PACS 100 & 100 & - & $18500 \pm 3500$ & $\mathrm{C}$ \\
PACS 160 & 160 & - & $24000 \pm 9600$ & $\mathrm{C}$ \\
SPIRE 250 & 250 & - & $20200 \pm 4500$ & $\mathrm{C}$ \\
SPIRE 350 & 350 & - & $17500 \pm 4700$ & $\mathrm{C}$ \\
SPIRE 500 & 500 & - & $<17200$ & $\mathrm{C}$ \\
\hline
\end{tabular}

Notes. Columns are the same as in Table D.1.

References. (A) Steinbring et al. (2002); (B) De Breuck et al. (2010); (C) Drouart et al. (2014).

Table D.8. Data for 4C $28.58(z=2.89)$.

\begin{tabular}{lcccc}
\hline \hline Filter & $\begin{array}{c}\lambda_{0} \\
{[\mu \mathrm{m}]}\end{array}$ & $\begin{array}{c}\text { Line } \\
{[\%]}\end{array}$ & $\begin{array}{c}\text { Flux } \\
{[\mu \mathrm{Jy}]}\end{array}$ & Ref. \\
\hline$F 439 W$ & 0.43 & - & $<0.84$ & $\mathrm{~A}$ \\
$F 675 W$ & 0.67 & - & $1.23 \pm 0.1$ & $\mathrm{~A}$ \\
$F 814 W$ & 0.83 & - & $0.92 \pm 0.1$ & $\mathrm{~A}$ \\
K(NIRC) & 2.2 & - & $13.1 \pm 1.3$ & $\mathrm{~B}$ \\
IRAC1 & 3.6 & - & $31.6 \pm 3.5$ & $\mathrm{C}$ \\
IRAC2 & 4.5 & - & $36.0 \pm 3.9$ & $\mathrm{C}$ \\
IRAC3 & 5.8 & - & $<62.6$ & $\mathrm{C}$ \\
IRAC4 & 8.0 & - & $40.9 \pm 4.4$ & $\mathrm{C}$ \\
IRS & 16.0 & - & $430.0 \pm 85.0$ & $\mathrm{C}$ \\
MIPS1 & 24.0 & - & $866.0 \pm 155.0$ & $\mathrm{C}$ \\
PACS 100 & 100 & - & $22800 \pm 2900$ & $\mathrm{D}$ \\
PACS 160 & 160 & - & $23600 \pm 8700$ & $\mathrm{D}$ \\
SPIRE 250 & 250 & - & $42500 \pm 4900$ & $\mathrm{D}$ \\
SPIRE 350 & 350 & - & $29700 \pm 5400$ & $\mathrm{D}$ \\
SPIRE 500 & 500 & - & $15500 \pm 4500$ & $\mathrm{D}$ \\
SCUBA 850 & 850.0 & - & $3930 \pm 950.0$ & $\mathrm{E}$ \\
\hline
\end{tabular}

Notes. Columns are the same as in Table D.1.

References. (A) Chambers et al. (1996b); (B) Akiyama et al. (2008); (C) De Breuck et al. (2010); (D) Drouart et al. (2014); (E) Archibald et al. (2001). 
G. Drouart et al.: Disentangling SF and AGN in luminous HzRGs

Table D.9. Data for USS 0943-242 $(z=2.92)$.

\begin{tabular}{lcccc}
\hline \hline Filter & $\begin{array}{c}\lambda_{0} \\
{[\mu \mathrm{m}]}\end{array}$ & $\begin{array}{c}\text { Line } \\
{[\%]}\end{array}$ & $\begin{array}{c}\text { Flux } \\
{[\mu \mathrm{Jy}]}\end{array}$ & Ref. \\
\hline$F 702 W$ & 0.7 & 11.0 & $3.58 \pm 0.3$ & $\mathrm{~A}$ \\
$F 160 W$ & 1.6 & - & $13.3 \pm 1.0$ & $\mathrm{~B}$ \\
IRAC1 & 3.6 & - & $21.5 \pm 2.6$ & $\mathrm{C}$ \\
IRAC2 & 4.5 & - & $28.4 \pm 3.2$ & $\mathrm{C}$ \\
IRAC3 & 5.8 & - & $<30.9$ & $\mathrm{C}$ \\
IRAC4 & 8.0 & - & $25.8 \pm 11.7$ & $\mathrm{C}$ \\
IRS & 16.0 & - & $170.0 \pm 48.0$ & $\mathrm{C}$ \\
MIPS1 & 24.0 & - & $518.0 \pm 40.0$ & $\mathrm{C}$ \\
MIPS2 & 70.0 & - & $<3390$ & $\mathrm{C}$ \\
MIPS3 & 160.0 & - & $<50900$ & $\mathrm{C}$ \\
PACS 100 & 100 & - & $<27600$ & $\mathrm{D}$ \\
PACS 160 & 170 & - & $23600 \pm 7700$ & $\mathrm{D}$ \\
SPIRE 250 & 250 & - & $25700 \pm 5200$ & $\mathrm{D}$ \\
SPIRE 350 & 350 & - & $31700 \pm 5500$ & $\mathrm{D}$ \\
SPIRE 500 & 500 & - & $35200 \pm 7300$ & $\mathrm{D}$ \\
1.3 mm (SEST) & 1300 & - & $<14200$ & $\mathrm{E}$ \\
\hline
\end{tabular}

Notes. Columns are the same as in Table D.1.

References. (A) Pentericci et al. (1999); (B) Pentericci et al. (2001); (C) De Breuck et al. (2010); (D) Drouart et al. (2014); (E) Cimatti et al. (1998).

Table D.10. Data for 4C $41.17(z=3.79)$.

\begin{tabular}{lcccc}
\hline \hline Filter & $\begin{array}{c}\lambda_{0} \\
{[\mu \mathrm{m}]}\end{array}$ & $\begin{array}{c}\text { Line } \\
{[\%]}\end{array}$ & $\begin{array}{c}\text { Flux } \\
{[\mu \mathrm{Jy}]}\end{array}$ & Ref. \\
\hline$F 702 W$ & 0.7 & - & $5.01 \pm 0.36$ & $\mathrm{~A}$ \\
$I$ (KPNO) & 0.9 & - & $4.5 \pm 1.5$ & $\mathrm{~B}$ \\
$J$ (NIRC) & 1.25 & - & $5.6 \pm 1.1$ & $\mathrm{C}$ \\
Ks(NIRC) & 2.155 & - & $13.6 \pm 2.8$ & $\mathrm{C}$ \\
IRAC1 & 3.6 & - & $23.4 \pm 2.40$ & $\mathrm{D}$ \\
IRAC2 & 4.5 & - & $27.5 \pm 2.80$ & $\mathrm{D}$ \\
IRAC3 & 5.8 & - & $35.6 \pm 3.70$ & $\mathrm{D}$ \\
IRAC4 & 8.0 & - & $36.5 \pm 3.50$ & $\mathrm{D}$ \\
IRS & 16.0 & - & $<181.0$ & $\mathrm{D}$ \\
MIPS1 & 24 & - & $370.0 \pm 40.0$ & $\mathrm{D}$ \\
PACS 70 & 70 & - & $<2600$ & $\mathrm{E}$ \\
PACS 160 & 170 & - & $16200 \pm 6700$ & $\mathrm{E}$ \\
SPIRE 250 & 250 & - & $35800 \pm 3500$ & $\mathrm{E}$ \\
SPIRE 350 & 350 & - & $43100 \pm 3700$ & $\mathrm{E}$ \\
SPIRE 500 & 500 & - & $38000 \pm 4500$ & $\mathrm{E}$ \\
UKT14 800 & 800 & - & $17400 \pm 3100$ & $\mathrm{~F}$ \\
SCUBA 850 & 850 & - & $12100 \pm 880$ & $\mathrm{G}$ \\
IRAM 1200 & 1200 & - & $4400 \pm 400$ & $\mathrm{H}$ \\
IRAM 1300 & 1300 & - & $2500 \pm 400$ & $\mathrm{I}$ \\
\hline
\end{tabular}

Notes. Columns are the same as in Table D.1.

References. (A) Miley et al. (1992); (B) Chambers et al. (1990); (C) van Breugel et al. (1998); (D) De Breuck et al. (2010), (E) Wylezalek et al. (2013b); (F) Dunlop et al. (1994); (G) Archibald et al. (2001); (H) Greve et al. (2007); (I) Chini \& Kruegel (1994).
Table D.11. Data for TN J2007-1316 $(z=3.84)$.

\begin{tabular}{lcccc}
\hline \hline Filter & $\begin{array}{c}\lambda_{0} \\
{[\mu \mathrm{m}]}\end{array}$ & $\begin{array}{c}\text { Line } \\
{[\%]}\end{array}$ & $\begin{array}{c}\text { Flux } \\
{[\mu \mathrm{Jy}]}\end{array}$ & Ref. \\
\hline$R$ & 0.65 & - & $2.06 \pm 0.4$ & $\mathrm{~A}$ \\
$I$ (CFHT) & 0.9 & - & $2.60 \pm 0.15$ & $\mathrm{~A}$ \\
$H$ (ISAAC) & 1.65 & - & $9.6 \pm 1.0$ & $\mathrm{~A}$ \\
$K$ (UKIRT) & 2.2 & - & $28.4 \pm 1.9$ & $\mathrm{~B}$ \\
IRAC1 & 3.6 & - & $46.6 \pm 4.8$ & $\mathrm{~A}$ \\
IRAC2 & 4.5 & - & $52.7 \pm 5.7$ & $\mathrm{~A}$ \\
IRAC3 & 5.8 & - & $<146.0$ & $\mathrm{~A}$ \\
IRAC4 & 8.0 & - & $135.1 \pm 16.9$ & $\mathrm{~A}$ \\
IRS & 16.0 & - & $378.0 \pm 113.0$ & $\mathrm{C}$ \\
MIPS1 & 24 & - & $385.0 \pm 40.0$ & $\mathrm{C}$ \\
PACS 100 & 105 & - & $<35025$ & $\mathrm{~A}$ \\
PACS 160 & 170 & - & $<62034$ & $\mathrm{~A}$ \\
SPIRE 250 & 250 & - & $13850 \pm 6100$ & $\mathrm{~A}$ \\
SPIRE 350 & 350 & - & $16500 \pm 6376$ & $\mathrm{~A}$ \\
SPIRE 500 & 500 & - & $7615 \pm 3322$ & $\mathrm{~A}$ \\
SCUBA 850 & 850 & - & $5800 \pm 1500$ & $\mathrm{D}$ \\
\hline
\end{tabular}

Notes. Columns are the same as in Table D.1

References. (A) Rocca-Volmerange et al. (2013); (B) Bornancini et al. (2007); (C) De Breuck et al. (2010); (D) Reuland et al. (2004). 


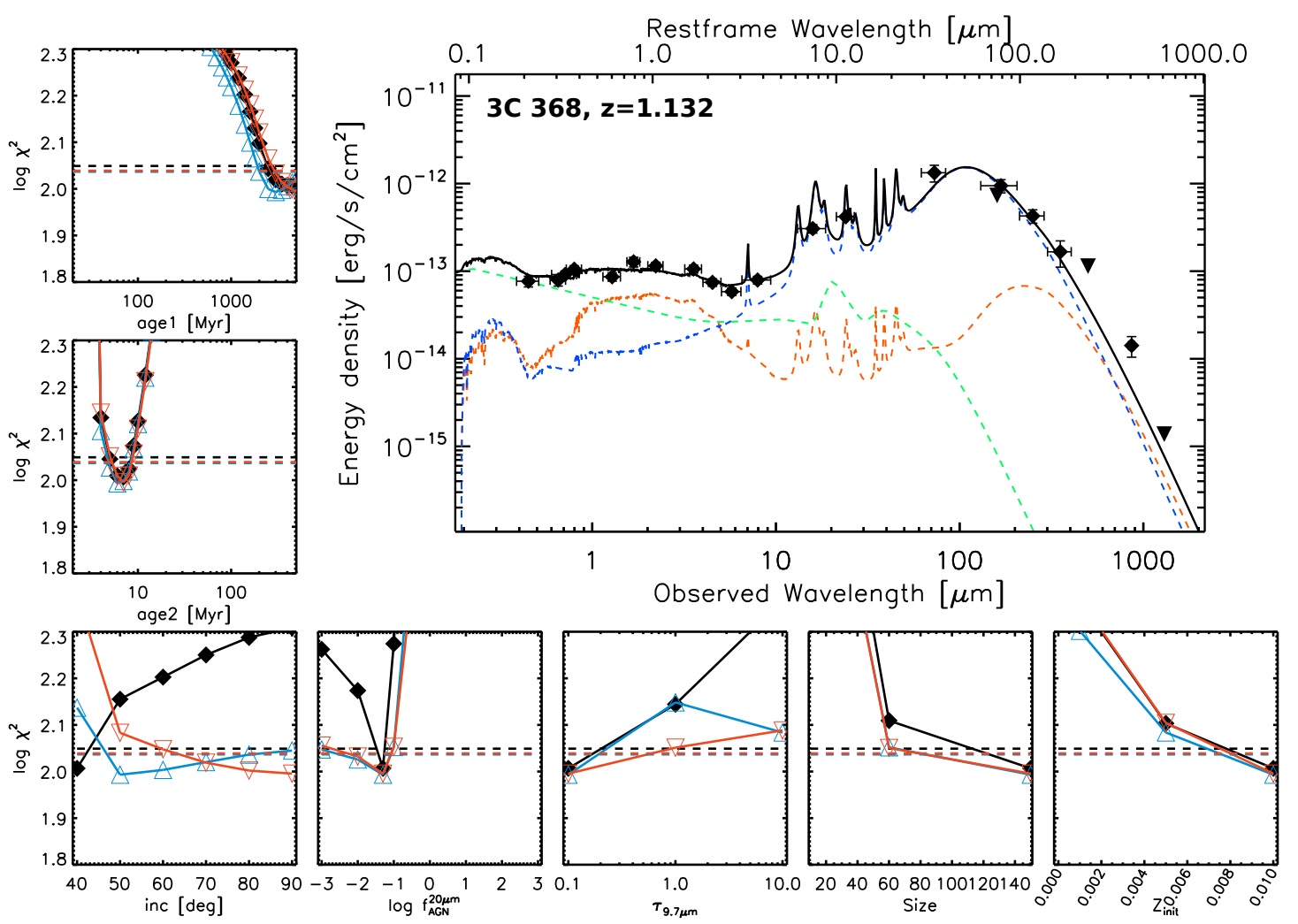

Fig. D.1. Best fit for 3C $368(z=1.132)$. The colour code and the layout are the same as in Fig. 2.
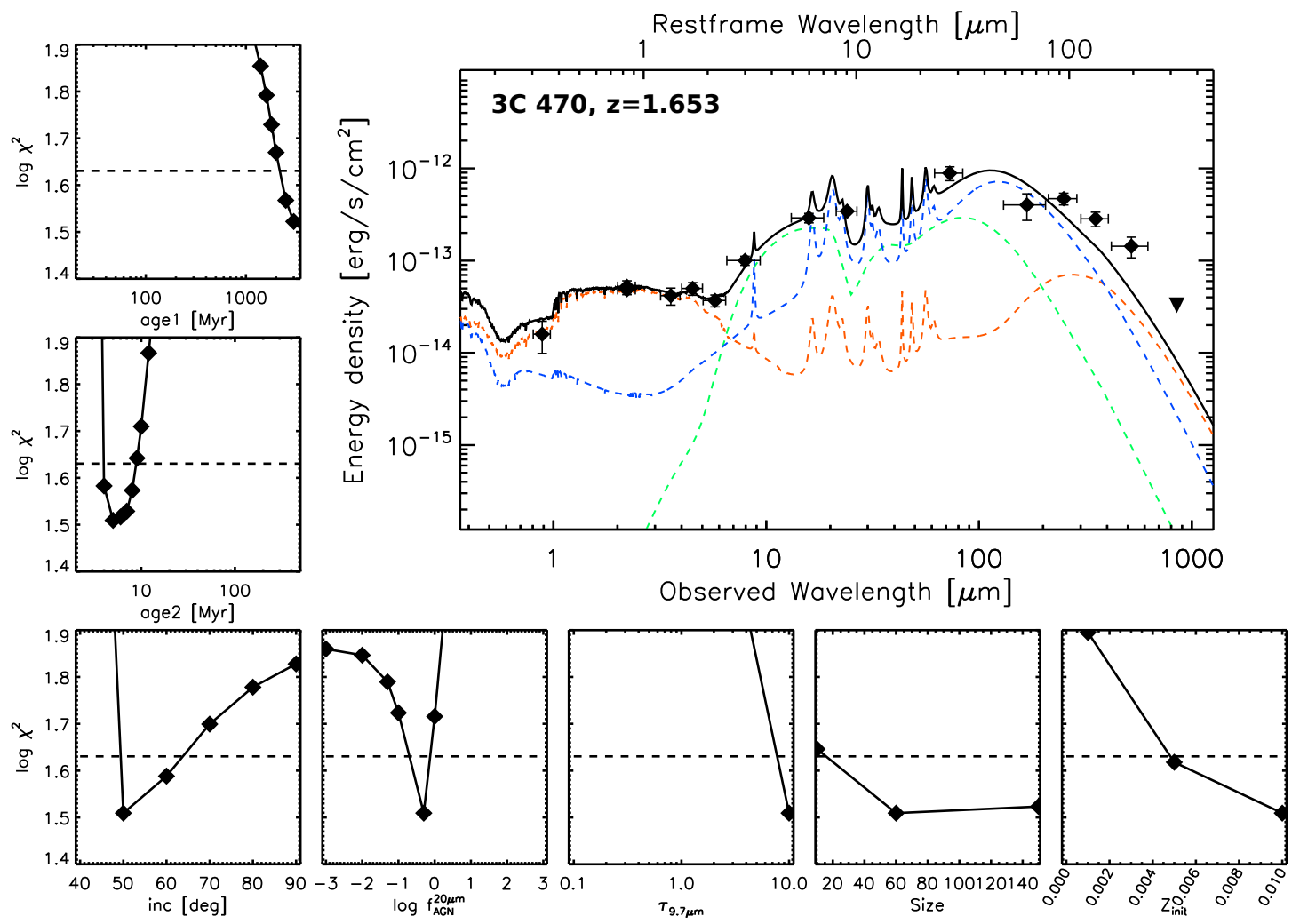

Fig. D.2. Best fit for 3C $470(z=1.653)$. The colour code and the layout are the same as in Fig. 2. No polarisation data are available for this galaxy, therefore the insets present the results of the without polarisation approach, reported in black. 
G. Drouart et al.: Disentangling SF and AGN in luminous HzRGs
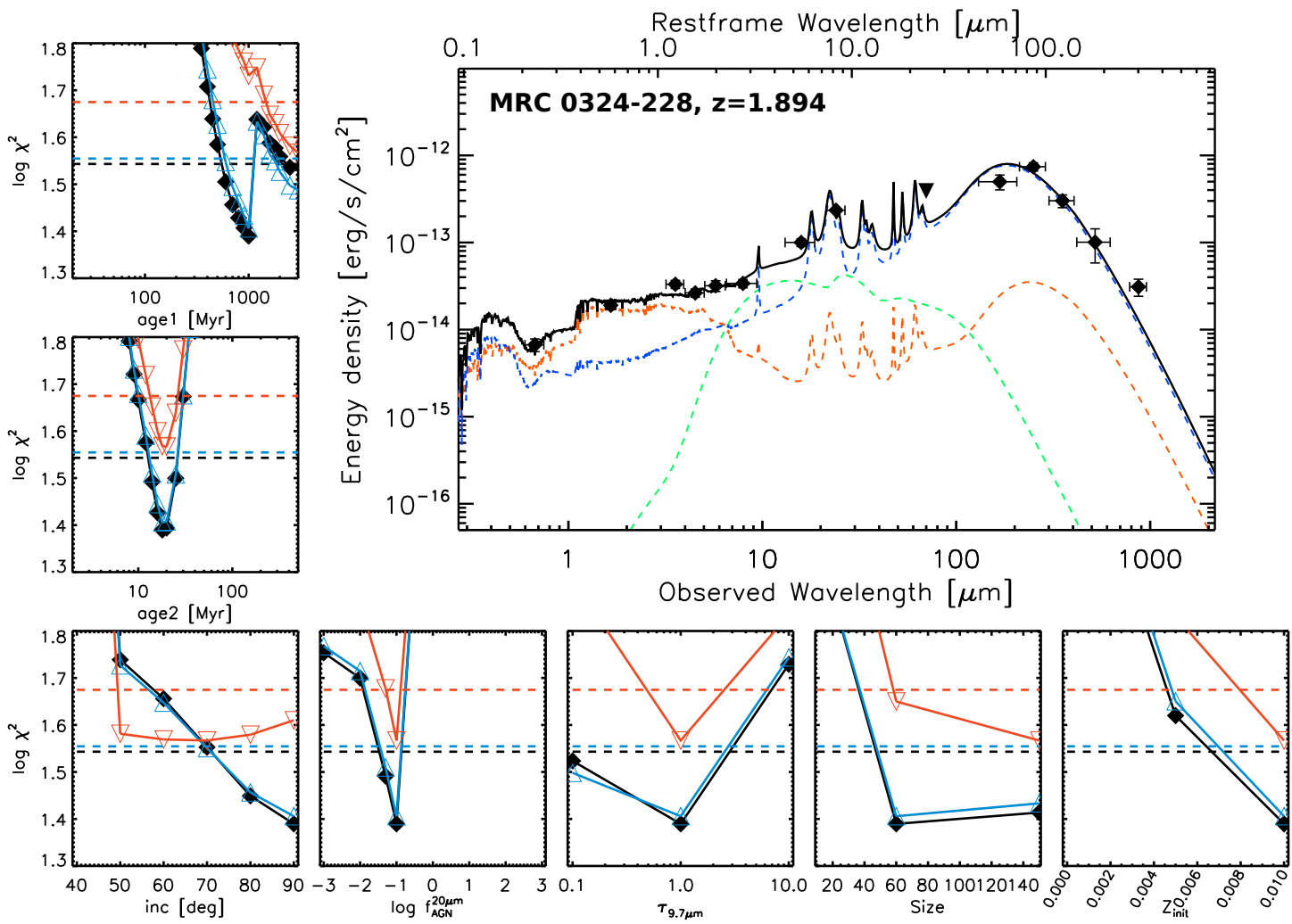

Fig. D.3. Best fit for MRC 0324-228 $(z=1.894)$. The colour code and the layout are the same as in Fig. 2.
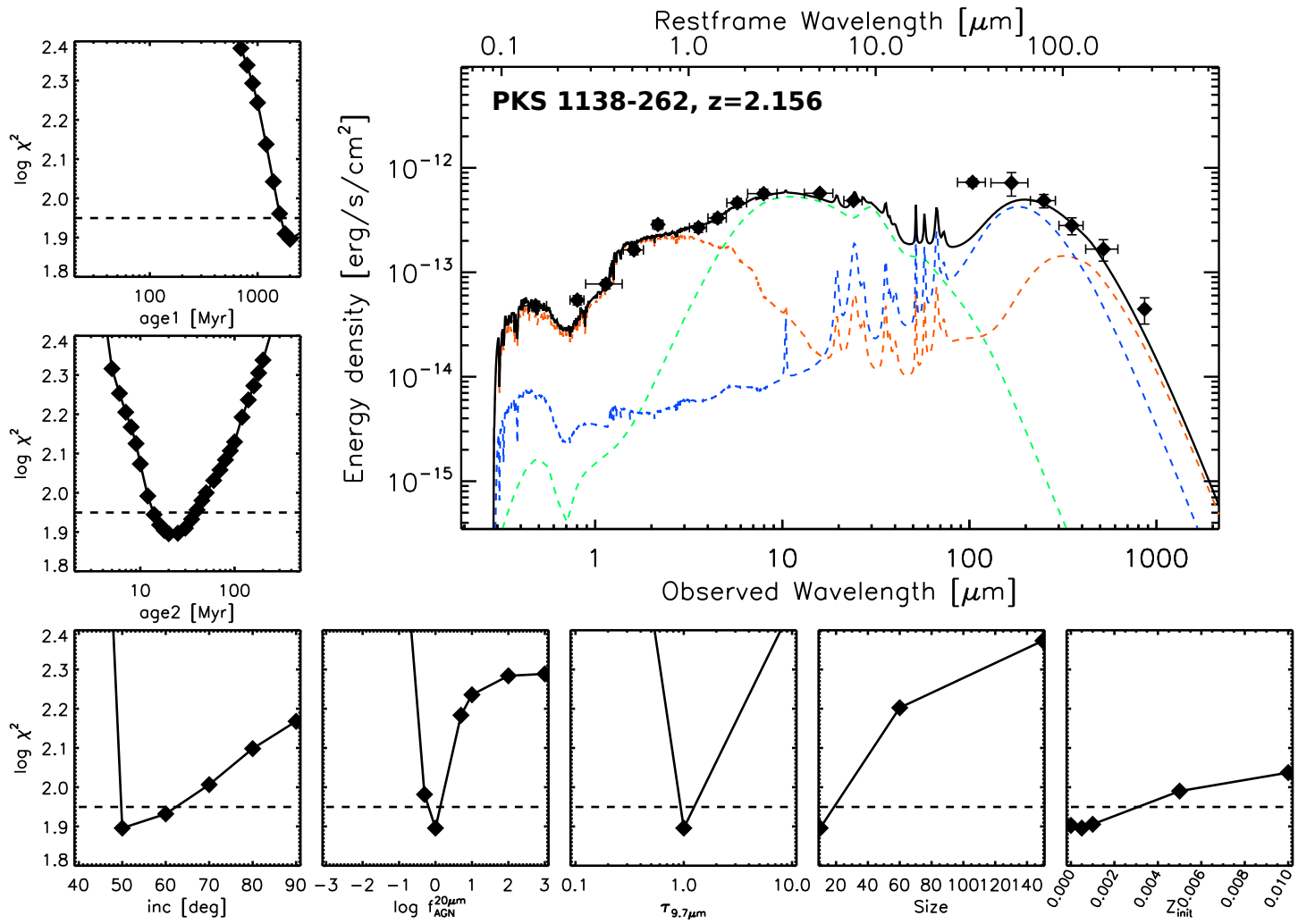

Fig. D.4. Best fit for PKS 1138-262 $(z=2.156)$. The colour code and the layout are the same as in Fig. 2. No polarisation data are available for this galaxy, therefore the insets present the results of the without polarisation approach, reported in black. 

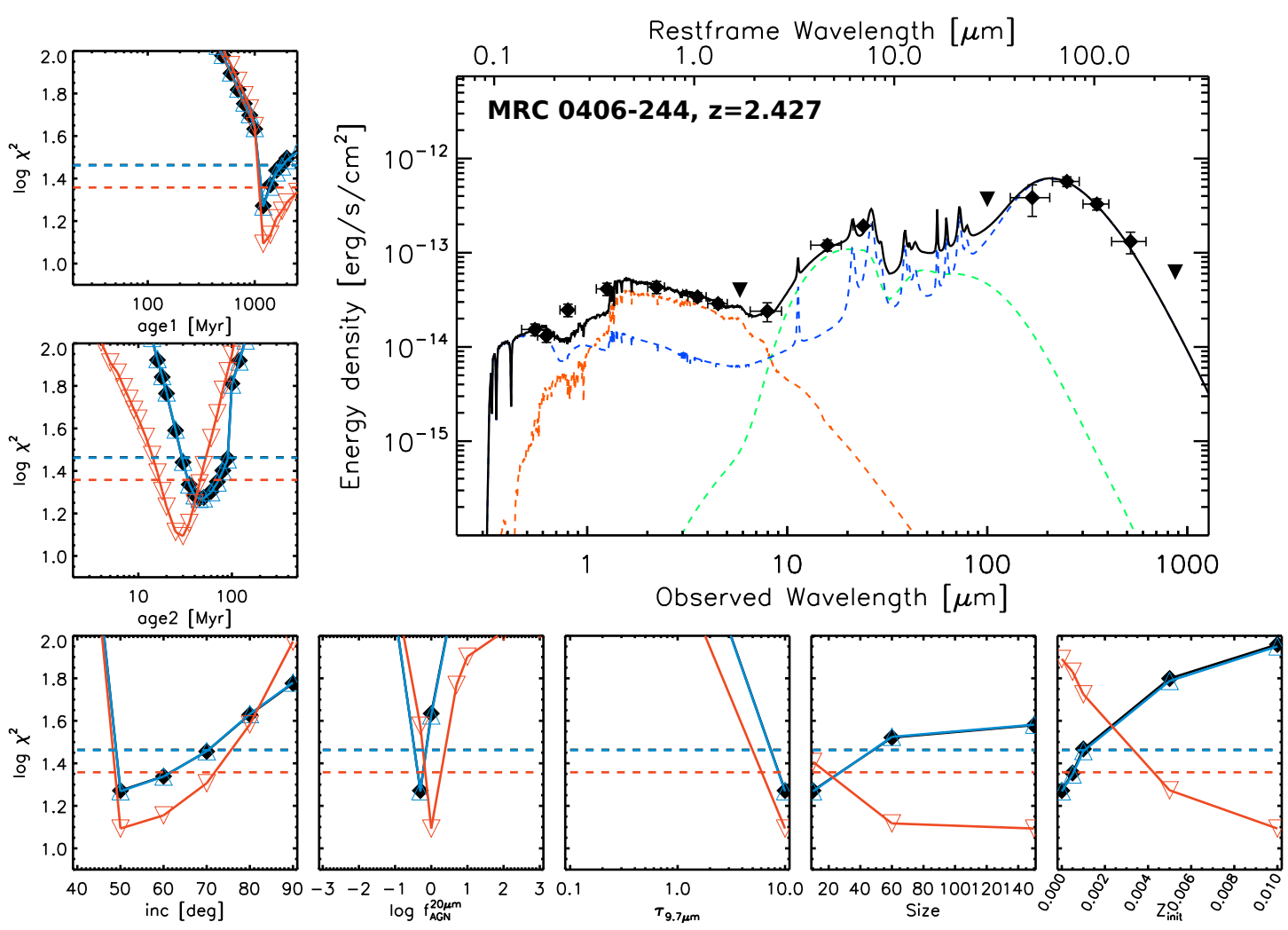

Fig. D.5. Best fit for MRC 0406-244 $(z=2.427)$. The colour code and the layout are the same as in Fig. 2.
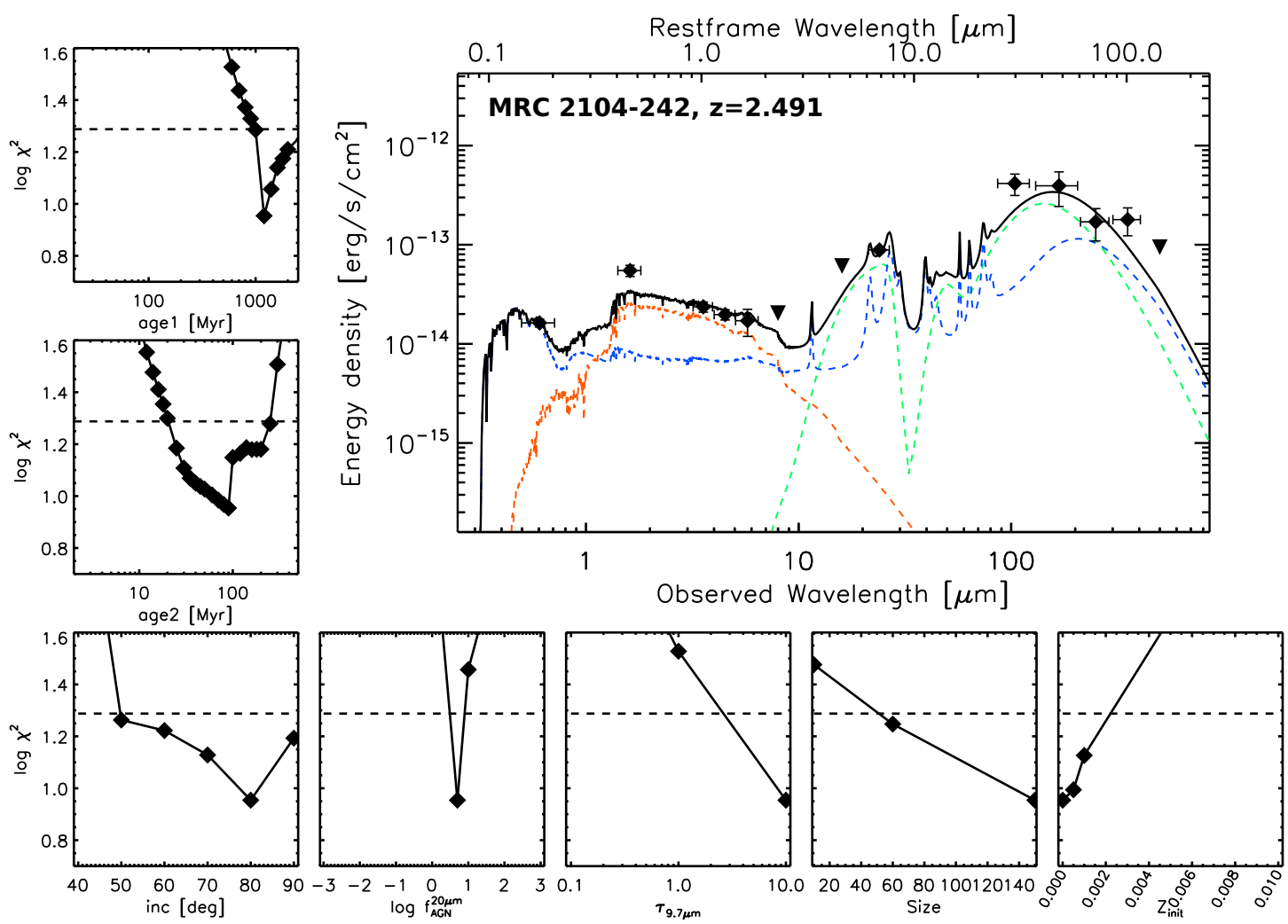

Fig. D.6. Best fit for MRC 2104-242 $(z=2.491)$. The colour code and the layout are the same as in Fig. 2. No polarisation data are available for this galaxy, therefore the insets present the results of the without polarisation approach, reported in black. 
G. Drouart et al.: Disentangling SF and AGN in luminous HzRGs
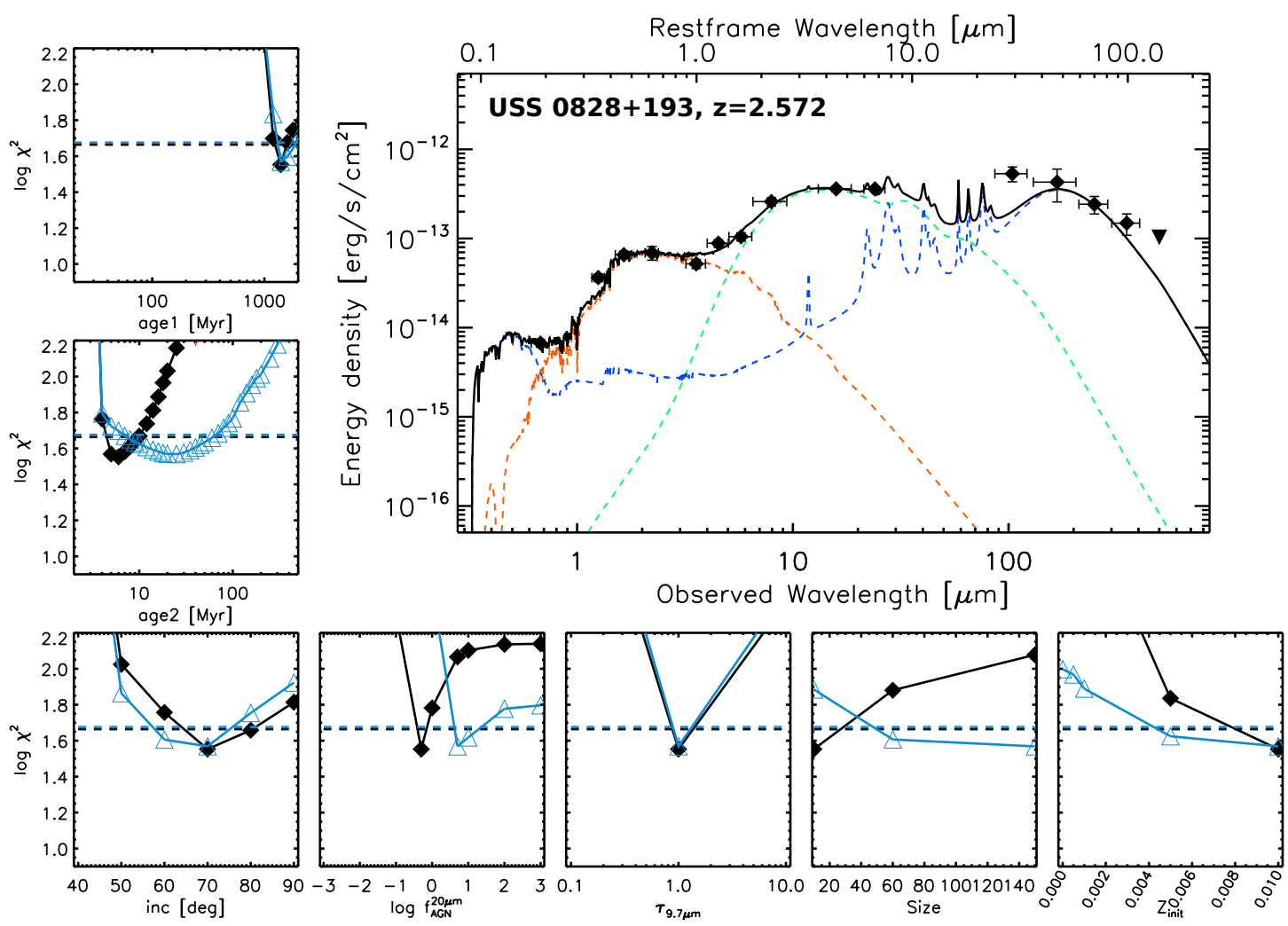

Fig. D.7. Best fit for USS $0828+193(z=2.572)$. The colour code and the layout are the same as in Fig. 2. Only the minimal subtraction case (blue lines) is reported for this source (see Sect. 4.4).
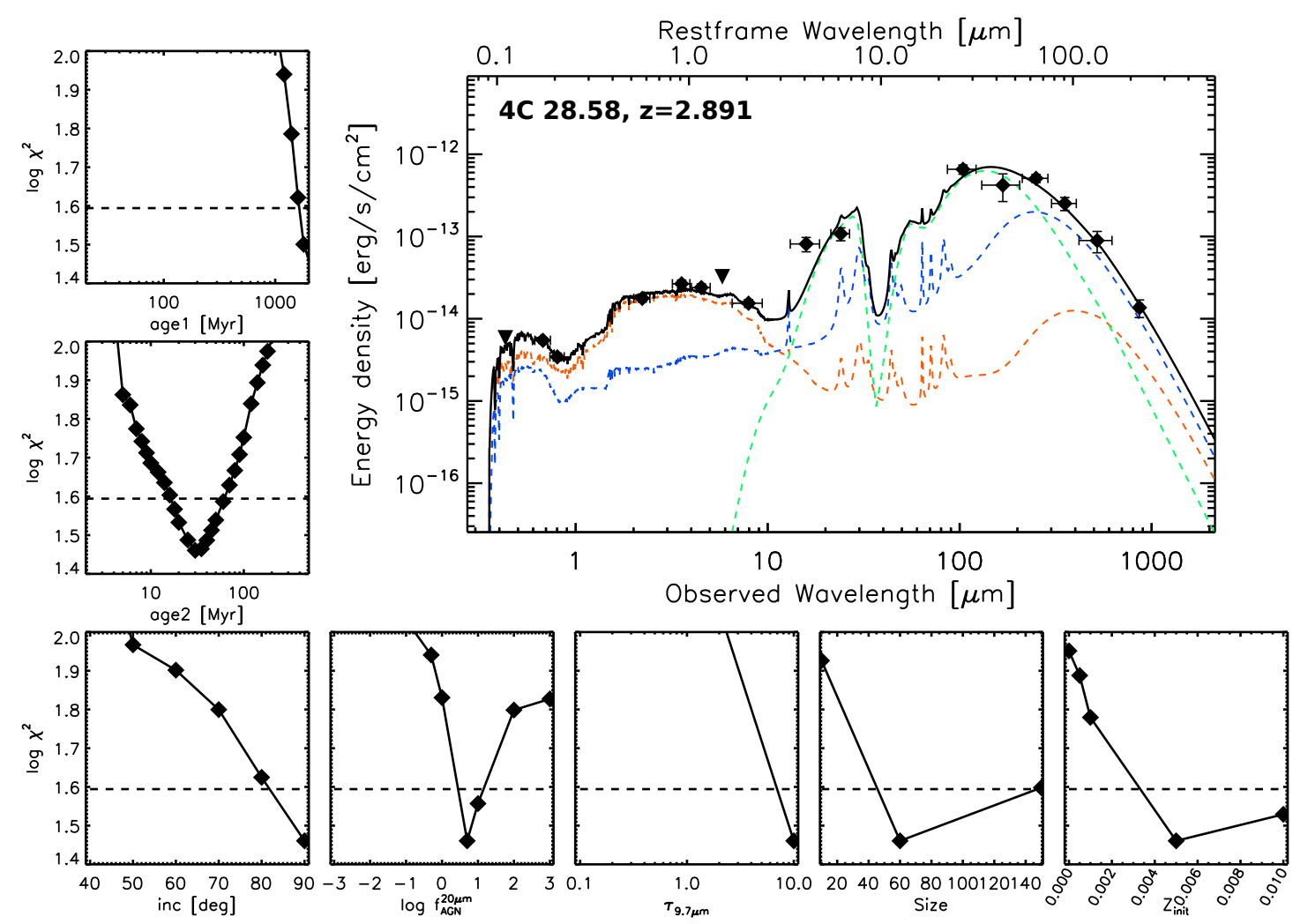

Fig. D.8. Best fit for 4C $28.58(z=2.891)$. The colour code and the layout are the same as in Fig. 2. No polarisation data are available for this galaxy, therefore the insets present the results of the withoutpolarisation approach, reported in black. 


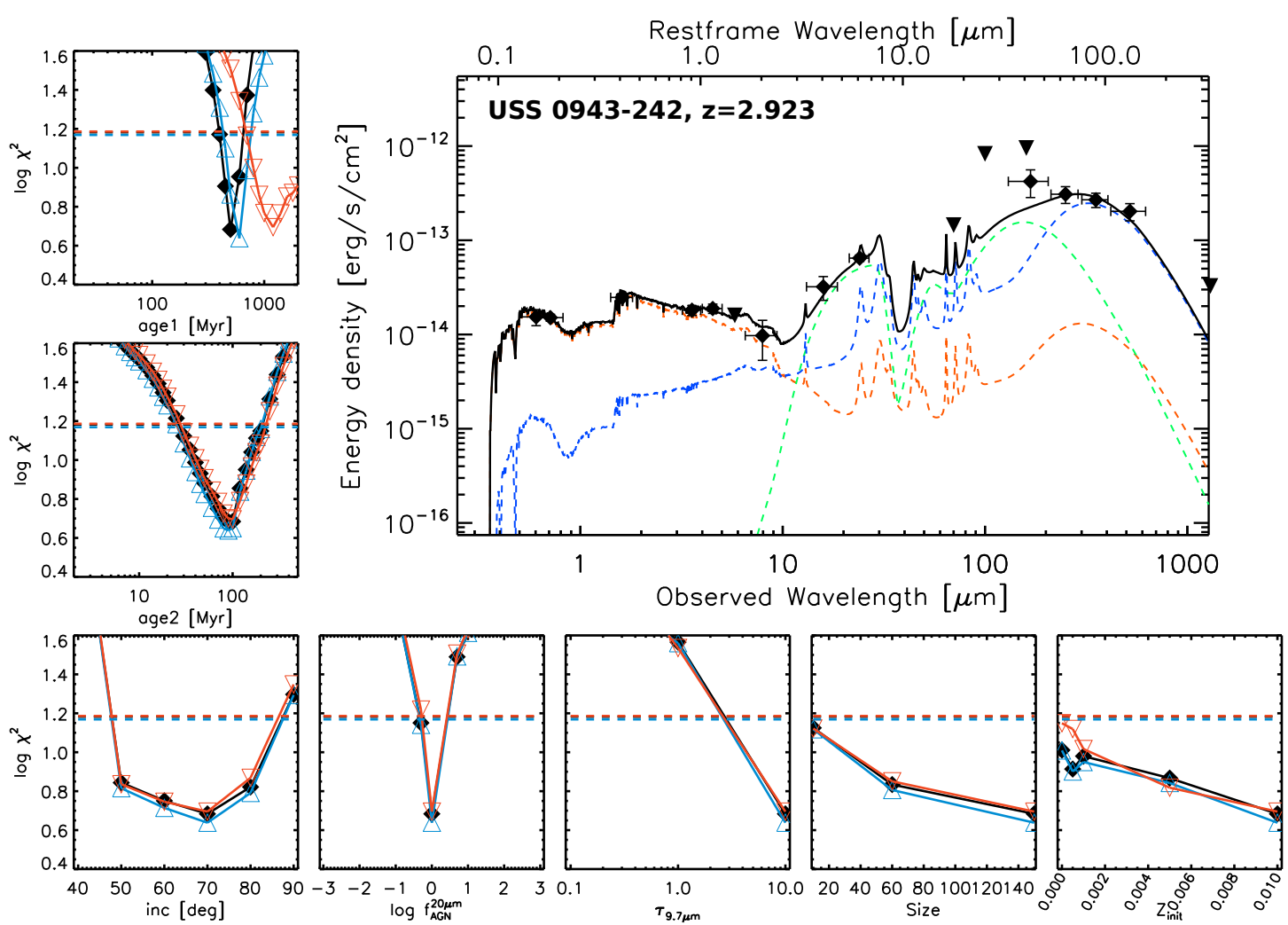

Fig. D.9. Best fit for USS 0943-242 $(z=2.923)$. The colour code and the layout are the same as in Fig. 2.

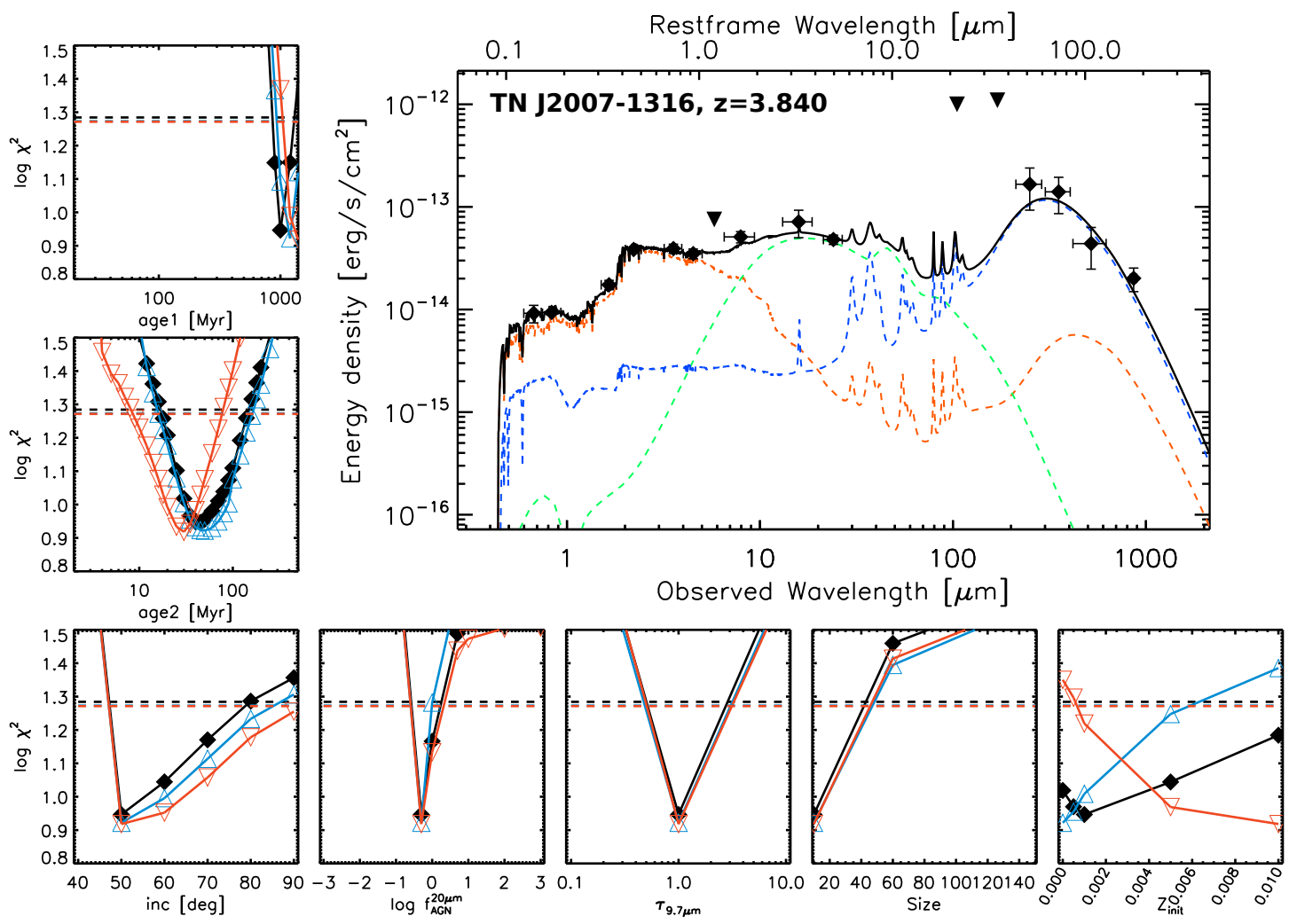

Fig. D.10. Best fit for TN J2007-1316 $(z=3.840)$. The colour code and the layout are the same as in Fig. 2. 